\title{
Kctd15 regulates nephron segment development by repressing Tfap2a activity
}

\author{
Brooke E. Chambers $^{1 *}$, Eleanor G. Clark ${ }^{1}$, Allison E. Gatz ${ }^{1}$, and Rebecca A. Wingert ${ }^{1 *}$ \\ ${ }^{1}$ Department of Biological Sciences, Center for Stem Cells and Regenerative Medicine, \\ Center for Zebrafish Research, University of Notre Dame, Notre Dame, 46556, USA
}

${ }^{*}$ Corresponding authors

Key Abbreviations: corpuscle of Stannius (CS); distal early (DE); distal late (DL); fluorescent whole mount in situ hybridization (FISH); immunofluorescence (IF); hours post fertilization (hpf); morpholino oligonucleotide (MO); Loop of Henle (LOH); potassium channel tetramerization domain 15 (kctd15); proximal straight tubule (PST); somite stage (ss); thick ascending limb (TAL); transcription factor AP-2 alpha (tfap2a); whole mount in situ hybridization (WISH); wild-type (WT)

\section{Correspondence:}

Rebecca A. Wingert, Ph.D., Department of Biological Sciences, University of Notre Dame, 100 Galvin Life Sciences, Notre Dame, IN 46556, USA; Email: rwingert@nd.edu, Phone: (574)-631-0907, Fax: (574)-631-741

Brooke E. Chambers, Department of Biological Sciences, University of Notre Dame, 100 Galvin Life Sciences, Notre Dame, IN 46556, USA; Email: bweaver4@nd.edu, Phone: (574)-631-0910, Fax: (574)631-7413 


\section{Abstract}

A functional vertebrate kidney relies on structural units called nephrons, which are epithelial tubules that contain a sequence of segments each expressing a distinct repertoire of solute transporters. To date, the transcriptional codes driving regional specification, solute transporter program activation, and terminal differentiation of segment populations remain poorly understood. We demonstrate for the first time that the KCTD15 paralogs, kctd15a and kctd15b, function in concert to restrict distal early $(\mathrm{DE}) /$ thick ascending limb ( $T A L)$ segment lineage assignment in the developing zebrafish pronephros by repressing Tfap2a activity. During renal ontogeny, expression of these factors co-localized with tfap2a in distal tubule precursors. kctd15 loss primed nephron cells to adopt distal fates by driving expansions in slc12a1, kcnj1a.1, and stc1 marker expression. These phenotypes were resultant of Tfap2a hyperactivity, where kctd15a/b-deficient embryos exhibited increased abundance of this transcription factor. Interestingly, tfap2a reciprocally promoted kctd15 transcription, unveiling a circuit of autoregulation operating in nephron progenitors. Concomitant kctd15b knockdown with tfap2a overexpression produced genetic synergy and further expanded the DE population. Our study provides

strong evidence that a transcription factor-repressor feedback module employs tight regulation of Tfap2a and Kctd15 kinetics to control nephron segment fate choice and differentiation during kidney development. 


\section{Introduction}

Mammalian kidney organogenesis is unique in that it entails three waves of assembly, the pronephros, the mesonephros, and the metanephros, where each successive version becomes more morphologically complex than the previous. ${ }^{1}$ Other vertebrates, such as fish and amphibians, undergo two phases of kidney development, the final form being the mesonephros. ${ }^{2}$ Importantly, each kidney version across these animal kingdoms shares a conserved structural unit, the nephron. Recently, aquatic animal models such as fish and frogs have surfaced as powerful organisms to study the fundamentals of nephron development. ${ }^{3}$

The nephron is the functional unit of the kidney and is comprised of three core components: a glomerulus, an epithelial tubule, and a collecting duct. The tubule is compartmentalized into a series of proximal, intermediate, and distal segments. A key gap in knowledge remains in understanding the developmental signals necessary for the differentiation of segment-specific nephron cell types. More specifically, the genetic regulation of Loop of Henle ( $\mathrm{LOH}$ ) formation remains highly understudied. The $\mathrm{LOH}$ is partitioned into three limbs and initiates a concentration gradient by transporting water, sodium chloride, and potassium. Zebrafish, being an aquatic species, lack the first two limbs of the LOH because water conservation is not physiologically requisite. However, zebrafish do possess a distal early (DE) segment which is analogous to the mammalian thick ascending limb (TAL) of the LOH, which express a conserved suite of solute transporters including the orthologues of SLC12A1, KCNJ1, and CLCNK. ${ }^{4,5}$ Defective TAL transporters are associated with neonatal and classical Bartter Syndrome, ion imbalance, polyuria, and renal failure. To this end, high-throughput screens in zebrafish have facilitated discovery of novel genes likely linked to renal tubular disorders. ${ }^{6,7}$ More recently, a forward genetic screen and subsequent mutant analysis identified transcription factor AP-2 alpha (tfap2a) as a keystone regulator of the DE/TAL terminal differentiation program. ${ }^{8}$ TFAP2A belongs to the AP-2 family of transcription factors, which generally function in an embryonic context to control proliferation and differentiation. ${ }^{9}$ 
Two Tfap2a gene regulatory network (GRN) candidates are zebrafish paralogs $k c t d 15 a$ and $k c t d 15 b$, which belong to the Potassium Channel Tetramerization Domain family. Proteins in this family harbor a conserved BTB/POZ (BR-C, ttk and bab/Pox virus and Zinc finger) protein-protein interaction motif situated at the N-terminus, however, demonstrate significant structural variability outside of this region. The BTB/POZ domain is essential for targeting proteins for ubiquitination and degradation. ${ }^{10} \mathrm{~A}$ diverse set of biological functions are assigned to these proteins such as transcriptional repression, gating of ion channels, regulating cytoskeletal elements, and acting as adaptor molecules. Mutations in KCTD genes can initiate human diseases such as breast cancer, medulloblastoma, epilepsy, pulmonary inflammation, and obesity highlighting the importance of further functional investigation. ${ }^{11}$ Humans, mice, and Xenopus possess one KCTD15 gene, however zebrafish acquired kctd15a and kctd15b paralogous versions due to an ancient species-specific genome duplication event. Zebrafish Kctd15a and Kctd15b amino acid residues exhibit a high degree of conservation with both splice variants of human KCTD15 protein and nearly identically alignment in the BTB/POZ functional motif (Figure S1).

In zebrafish, $k c t d 15 a / b$ inhibit neural crest development by two distinct modules: attenuation of canonical Wnt signaling and direct repression of Tfap2a. KCTD15 strongly inhibits TFAP2A by binding to its proline-rich activation domain. ${ }^{12,13} \mathrm{Kctd} 15$ is a substrate for SUMOylation; this post-translational modification is associated with transcriptional repression. However, previous studies suggest nonSumoylated Kctd15 functions during neural crest formation. ${ }^{14}$ Genome wide association studies have revealed connections between Kctd15 and AP-2 to metabolic conditions such as obesity, diabetes, and eating disorders. ${ }^{15,16}$ Contrary to neural crest development, in Drosophila, KCTD15 facilitates SUMOylation of Tfap2b to regulate consummatory behavior and repress the transduction of adipogenesis and insulin signaling pathways. ${ }^{17,18}$ These findings authenticate the need for tissuespecific interrogation of Kctd15 function. Here, we demonstrate zebrafish $k c t d 15$ paralogs are novel regulators of nephron segment commitment and inhibit DE/TAL differentiation by participating in repressor-mediated genetic feedback with tfap2a. 


\section{Methods}

\section{Ethics statement and zebrafish husbandry}

Adult zebrafish were maintained at the University of Notre Dame Freimann Life Science Center. All studies were supervised by the University of Notre Dame Institutional Animal Care and Use Committee (IACUC), under protocol numbers 13-021 and 16-025. All WT experiments were conducted with the Tübingen strain. Embryonic zebrafish were incubated in E3 medium, staged and fixed as previously described. ${ }^{19,20}$

\section{Whole-mount and fluorescent in situ hybridization (WISH, FISH)}

WISH and FISH were performed as previously described. ${ }^{21}$ Digoxigenin and Fluorescein anti-sense RNA probes were synthesized by T7, T3, or SP6 in vitro transcription (Roche Diagnostics) from linearized IMAGE clone plasmids. Digoxigenin-labeled probes included: kctd15a, kctd15b, kcnj1a.1, slc12a1, stc1, slc12a3, and trpm7. Fluorescein-labeled probes included: tfap2a, slc12a3, slc12a1, kcnj1a.1, cdh17. Gene expression studies were performed in triplicate with sample size of $n>20$ for each replicate. Representative samples from each experimental group were imaged and analyzed.

\section{Whole-mount immunofluorescence (IF)}

Whole mount IF was completed as previously described. ${ }^{21}$ For all IF experiments, embryos were fixed in freshly diluted 4\% PFA for 2 hours at room temperature. 32\% EM Grade PFA (Electron Microscopy Sciences, 15714) was diluted to $4 \%$ concentration in 1X PBS. After fixation, embryos were stored in $100 \% \mathrm{MeOH}$ at $-20^{\circ} \mathrm{C}$ for future use. Primary antibody dilutions consisted of anti-laminin (1:100; SigmaAldrich, L9393), anti-T4 supernatant (1:200; Developmental Studies Hybridoma Bank, Na-K-Cl cotransporter, AB_528406), and anti-Tfap2a (1:50; LifeSpan Biosciences, LS-C87212-100). Secondary antibodies (1:500) include goat anti-rabbit, rabbit anti-mouse, and donkey anti-goat (Invitrogen: A11034, A11061, A11055). 4,6-diamidino-2-phenylindole dihydrochloride (DAPI; Invitrogen, D1306) was used for nuclear staining. 


\section{Morpholino knockdown and RT-PCR}

Morpholino oligonucleotides (MO) were synthesized by GeneTools, LLC. MOs were solubilized in DNase/RNase-free water to make $4 \mathrm{mM}$ stock concentrations and stored at $-20^{\circ} \mathrm{C}$. Splice-blocking MOs for $k c t d 15 a$ and $k c t d 15 b$ genes were designed to target the exon1-intron1 splice sites, and microinjected at dosages of $1 \mathrm{ng}$ and $3 \mathrm{ng}$, respectively. MO efficacy was validated by RT-PCR. RNA was extracted from pools of 20 embryos, cDNA was synthesized using random hexamers (Superscript IV, Invitrogen), and PCR was performed to amplify target site. Products were ran on a $1.5 \%$ Agarose gel, extracted, and sequenced. See Table S1 for specific MO and primer sequences.

\section{gRNA design and crispant generation}

gRNAs targeting the BTB-POZ encoding regions in kctd15a and $k c t d 15 b$ were designed using the CHOPCHOP web-based tool (https://chopchop.cbu.uib.no/). kctd15a sgRNA1 and sgRNA2 targeted different regions in exon 3. kctd15b sgRNA1 and sgRNA2 targeted different regions in exon 1. gRNA templates were annealed to a constant oligonucleotide as previously described, ${ }^{22}$ and RNA was synthesized using the T7 Megascript kit (Ambion). Multiplexed gene editing was achieved by injecting a cocktail containing all four gRNAs. Microinjection mix was prepared by combining gRNAs (60 ng/ $\mathrm{LL})$ and Cas9 protein $(0.8 \mu \mathrm{M})$ followed by incubation for 5 minutes at $37^{\circ} \mathrm{C}$. Embryos were injected at the one-cell stage with $\sim 5 \mathrm{~nL}$ of injection mix. T7 endonuclease assay was used to confirm genome editing. For $k c t d 15 a$ and $k c t d 15 b$ crispant verification, primers were designed to flank both sgRNA target sites in exons 3 and 1 respectively. In short, DNA was prepared from individual animals and Accuprime Pfx SuperMix (Invitrogen) was used to amplify target sites. PCR products were column purified with QIAquick PCR purification kit (Qiagen). $300 \mathrm{ng}$ of purified product and $2 \mu \mathrm{L} 10 \mathrm{x}$ NEB Buffer 2 (total volume $20 \mu \mathrm{L}$ ) was rehybridized in a thermocycler using the following program: 5 min at $95^{\circ} \mathrm{C}, \operatorname{ramp}$ down to $85^{\circ} \mathrm{C}$ at $2^{\circ} \mathrm{C} / \mathrm{s}$, ramp down to $25^{\circ} \mathrm{C}$ at $0.1^{\circ} \mathrm{C} / \mathrm{s}, 25^{\circ} \mathrm{C}$ for $10 \mathrm{~min}$. Rehybridized product was 
digested with $1 \mu \mathrm{L}$ T7 endonuclease I enzyme (New England Biolabs) at $37^{\circ} \mathrm{C}$ for 1 hour and separated on a $1.5 \%$ agarose gel. See Table S1 for specific gRNA and primer sequences.

\section{Overexpression experiments}

A kctd15a pCS2 construct was designed with Not1 and Xhol flanking the open reading frame allowing for in vitro synthesis of full-length sense cRNA using an SP6 mMessage Machine kit (Ambion). 60 pg of kctd15a cRNA was injected into one-cell stage embryos for overexpression. For tfap2a overexpression experiments, the $h$ s:tfap2a transgenic $\left[\operatorname{Tg}(h s p 70: t f a p 2 a)^{\mathrm{x} 24}\right]$, which was a generous gift from Bruce Riley (Texas A\&M University, USA), was employed. To activate the heat-shock inducible tfap2a transgene, embryos were incubated at $38^{\circ} \mathrm{C}$ for 30 minutes at the $8 \mathrm{ss}$ or $12 \mathrm{ss} . .^{23,24}$ Genotyping for the hs:tfap2a allele was performed as previously described. ${ }^{8}$ See Table S1 for genotyping primers.

\section{Image Acquisition and statistical analysis}

WISH samples were imaged with a Nikon Eclipse Ni with DS-Fi2 camera. FISH and IF samples were imaged with a Nikon C2 confocal microscope. The Nikon elements imaging software polyline tool was employed to quantify absolute lengths of gene expression domains. Confocal z-stacks were processed with Fiji (Image J) software and the plot profile feature was used to collect fluorescent intensity values. All graphical and statistical analyses were completed with GraphPad Prism. A minimum of three samples from control and experimental groups were imaged and scored. Unpaired t-test tests and oneway ANOVA analyses were conducted as appropriate and mean \pm standard deviation was reported. 


\section{Results}

\section{kctd15a and $k c t d 15 b$ are expressed in the developing pronephros}

Zebrafish and frog studies have previously reported kctd15 transcript expression in the pronephros. ${ }^{25,26}$ Data from the Genitourinary Development Molecular Anatomy Project (GUDMAP) detected KCTD15 expression in nascent mammalian nephrons. Microarray results indicated upregulated Kctd15 expression in developing renal vesicles in E12.5 mice (RID:Q-6PTG). Single cell RNA-sequencing of a week 17 human kidney cortex revealed elevated KCTD15 expression clustered with the developing nephron populace (RID:16-5HBT). ${ }^{27,28}$ This documented expression positions $k c t d 15 a$ and $k c t d 15 b$ as excellent candidates of nephron regulation. To expand on these previous studies, we assembled a comprehensive time course of kctd15a and kctd15b spatial expression over the span of kidney development using the genetically tractable zebrafish pronephros model. Upon WISH analysis of kctd15a and kctd15b in wild-type (WT) animals, transcripts were first detectable in the intermediate mesoderm (IM) at the 10 somite stage (ss) (Figure 1A). Throughout the duration of pronephric development, kctd15a and kctd15b showed similar expression patterns becoming increasingly restricted to the distal nephron progenitor domain (Figure 1B). Because the expression patterns of these factors in the developing kidney show minimal deviation from one another, it is highly possible they function redundantly in this context.

Next, we wanted to determine how the $k c t d 15 a$ and $k c t d 15 b$ expression domains spatially correlate to tfap2a transcripts in the developing pronephros, since these factors repress Tfap2a in neural crest. $^{12,25}$ Using fluorescent in situ hybridization (FISH), kctd15a transcripts were found to be co-expressed throughout the tfap2a+ IM populace at the 12 ss in WT embryos. (Figure 1C). At 24 hours post fertilization (hpf), kctd15a and $k c t d 15 b$ were expressed throughout the $t f a p 2 a^{+}$pronephric domain (Figure 1D,E). These experiments demonstrate $k c t d 15 a$ and $k c t d 15 b$ are expressed in developing distal precursors and their localization largely overlaps with tfap2a at multiple timepoints during development. 


\section{$k c t d 15 a / b$ regulates the expression of distal nephron markers}

In zebrafish, $k c t d 15 a / b$ deficiency leads to an expansion of neural crest fate ${ }^{25,29}$ most likely due to the inability to repress Tfap2a. Because $k c t d 15 a / b$ function had never been studied in the context of kidney organogenesis, we developed loss and gain of function strategies to assess their involvement in nephrogenesis. Knockdown of kctd15a and kctd15b was achieved by injecting morpholinos to disrupt splicing between the exon 1 and exon 2 junctions, and validated by RT-PCR analyses (Figure S2). For a parallel independent loss of function model, we genetically engineered F0 kctd15a, kctd15b, and kctd15a/b CRISPR mutants (crispants) by multiplexing sgRNAs targeting different regions of the BTB/POZ functional domain to induce biallelic disruptions (Figure S3). T7 endonuclease assays and Sanger sequencing confirmed successful genome editing (Figure S3). kctd15a/b morphants, kctd15a crispants, and kctd15b crispants developed pericardial edema and increased dorsal head pigmentation by 48 hpf consistent with other live phenotype reports (Figure S2, S3). ${ }^{25,29}$

Because kctd15 transcripts localized to the developing pandistal pronephric region (Figure 1), we first decided to survey for alterations in DE and DL marker expression, which are analogous to the mammalian TAL and distal convoluted tubule (DCT), respectively. $k c t d 15 a, k c t d 15 b$, and $k c t d 15 a / b$ morphants and crispants exhibited drastic expansions of the DE markers kcnj1a.1 and slc12a1 as compared to WT controls (Figure 2A-D). In each kctd15-deficient state, slc12a $1^{+}$cells encroached the DL territory marked by s/c12a3 (Figure 2C). Notably, previous study indicated when tfap2a is overexpressed, an expansion of DE solute transporter expression occurs. ${ }^{8}$ These parallel phenotypes highlight the possibility that this alteration of distal nephron fate could be due to inadequate repression of Tfap2a protein by Kctd15a/b. Generally, kctd15 crispants manifested slightly milder DE phenotypes as compared to kctd15 morphants, most likely because F0 crispant cellular composition is mosaic in nature and generates a mix of homozygous and heterozygous mutants. Nevertheless, $k c t d 15$ crispants recapitulated kctd15 morphant distal nephron phenotypes corroborating the use of MO mediated 
knockdown in subsequent experiments. As expected, compound MO knockdown of kctd15a and $k c t d 15 b$ elicited the most severe expansions of DE marker expression, suggesting that these paralogs evolved redundant functions in teleosts (Figure 2B, 2D). Overexpression of $k c t d 15 a$ cRNA produced a significantly decreased DE in conjunction with an expanded DL segment (Figure 2A-D). kctd15a overexpression also caused a substantial decline in kcnj1a. $1^{+}$ionocyte number (Figure S4). Matching phenotypes occurred upon kctd15b overexpression (data not shown). Next, we assessed S/c12a1 protein expression in $k c t d 15 a / b$ morphants. We found that $k c t d 15 a / b$ morphants exhibit a significant expansion of the S/c12a1 protein domain compared to WT embryos (Figure 2E). Generation of fluorescent intensity profiles confirmed a significant elevation in S/c12a1 signal in $k c t d 15 a / b$-deficient embryos compared to WT controls (Figure 2E-H). Quantification of cell number found that $k c t d 15 a / b$ morphants have nearly double the number of S/c12a $1^{+}$cells (Figure 2I).

Further, we examined a distal nephron associated cell type, the corpuscle of Stannius (CS). Briefly, the CS is an aggregate of cells situated between the DE and DL segments that eventually bud off the pronephric tubule to form an endocrine gland that maintains calcium homeostasis. ${ }^{30} \mathrm{~A}$ recent study illustrated that CS cells transdifferentiate from the DE segment by a gland extrusion mechanism. ${ }^{31}$ $k c t d 15 a, k c t d 15 b$, and $k c t d 15 a / b$ morphants and crispants all possess elevated numbers of CS cells as marked by stc1 compared to WTs (Figure 3A,B). Surprisingly, we noticed ectopic stc ${ }^{+}$cells formed at the proximal end of the DE domain that were clearly separate from the principal CS cell cluster in $k c t d 15 a / b$ morphant embryos (Figure 3A). Upon closer examination, we discovered kctd15a/b morphants undergo premature CS gland extrusion at $24 \mathrm{hpf}$, as this group of cells formed a basement membrane and separated from the pronephric tubule (Figure 3C). As tfap2a deficiency affects CS differentiation, it is not surprising that disrupting the balance of $k c t d 15 \mathrm{a} / \mathrm{b}$ alters this process as well. ${ }^{8}$ Collectively, our genetic experiments revealed alterations in DE, DL, and CS cell differentiation, identifying $k c t d 15$ as a novel regulator of distal nephron identity. 


\section{$k c t d 15 a / b$ depletion initiates ectopic DE formation in flanking pronephros segments}

Next, we decided to examine the effect of dual $k c t d 15 a / b$ knockdown on the neighboring nephron segment populations, as zebrafish $k c t d 15 a$ and $k c t d 15 b$ share over 90 percent amino acid sequence identity and likely have overlapping functions (Figure S1). We employed FISH to visualize the DE and DL nephron segment domains marked by kcnj1a.1 and s/c12a3 respectively. In 24 hpf WT animals, there is a sharp separation between the DE and DL segment compartments (Figure 4A,B). Conversely, in kctd15a/b morphants, kcnj1a. $1^{+}$cells were located in the DL segment. This significant overlap of DE and DL segment identities was made evident upon generation of a fluorescent intensity plot depicting a representative $k c t d 15 a / b$ morphant (Figure 4C). A portion of these cells dually expressed kcnj1a.1 and slc12a3, suggesting segment fate infidelity (Figure 4A). This dual marker expression is highly reminiscent of the resultant phenotype triggered by overexpression of tfap $2 a{ }^{8}$ Absolute length quantification of slc12a3 revealed that the $k c t d 15 a / b$ morphant DL domain is significantly shorter than WT controls (Figure 4D). These data indicate that $k c t d 15 a / b$ deficiency results in an expansion of the DE lineage at the expense of the DL. To probe the rostral DE boundary and its adjacent proximal straight tubule (PST) segment, trpm7 expression was assessed. WT animals have a well-defined DE/PST boundary (Figure 4E,F). Contrary to this, kctd15a/b morphant DE cells occupied the $\operatorname{trpm} 7^{+}$ PST region (Figure 4E,G). Additionally, some cells co-expressed kcnj1a.1 and trpm7, indicating $k c t d 15 a / b$-deficiency was potent enough to induce proximal nephron cells to express a distal nephron signature gene. Further, the absolute length of the PST was significantly reduced in $k c t d 15 a / b$ morphants (Figure $4 \mathrm{H}$ ). Taken together, these data suggest that $k c t d 15 a / b$ factors function to suppress DE lineage in neighboring segment populations during nephrogenesis. 


\section{kctd15a/b knockdown expands Tfap2a expression in the developing kidney}

To evaluate if $k c t d 15 a / b$ are regulating nephron differentiation by affecting Tfap2a localization or stability, we performed IF experiments on $24 \mathrm{hpf} k c t d 15 a / b$ morphants. $k c t d 15 a / b$ morphants showed expanded pronephric expression of Tfap2a protein compared to WTs (Figure 5A). Specifically, this morphant expansion was characterized by residual proximal nephron Tfap2a protein expression. This greatly contrasts WTs that exhibit an abrupt cutoff of Tfap2a protein expression at the proximal limit of the Slc $12 a 1^{+}$DE (Figure 5A). Generation of fluorescent intensity profiles revealed differentially elevated morphant Tfap2a expression that was particularly evident within a $100-220 \mu \mathrm{m}$ region that maps to the DE territory (Figure 5B). kctd15a/b morphants also exhibited a significant elevation of Tfap2a fluorescent signal within the 100-220 $\mu \mathrm{m}$ zone (Figure 5C,D). Further, $k c t d 15 a / b$ morphants exhibited a statistically significant increase in Tfap2a ${ }^{+}$pronephric nuclei than WT controls (Figure 5E). In summary, these results indicate that when $k c t d 15 a / b$ repressors are absent, the number of Tfap2a expressing cells increases in the pronephros and simultaneously parallels an expansion of the SIc12a $1^{+} \mathrm{DE}$. Our data illustrating alterations in pronephric Tfap2a protein expression reveals a new mechanistic layer that is distinct from previous in vitro studies, which found nuclear extracts exhibited no changes in Tfap2a protein abundance in the absence of Kctd15 and the main mode of inhibition was executed by direct binding to the Tfap2a transactivation domain. ${ }^{12}$ Whole-mount IF allowed for tracking cellular changes in Tfap2a protein expression in specific nephron regions. Based on these IF results, we hypothesized that expanded Tfap2a pronephric expression is the root cause of the DE lineage alterations that occur in $k c t d 15 a / b$ morphants.

\section{$k c t d 15 a / b$ and tfap2a engage in genetic feedback circuitry}

Because we discovered heightened Tfap2a protein signal in the pronephros in response to $k c t d 15 a / b$ deficiency (Figure 5), we postulated a genetic feedback mechanism might be responsible for this phenotype. In support of this concept, a previous study reported $k c t d 15 a / b$ zebrafish mutants manifest 
upregulated tfap2a neural expression at the 8-9 ss. ${ }^{29}$ To probe potential genetic feedback processes, we performed $\mathrm{FISH}$ at the 10 ss to detect fluctuations in tfap2a transcript expression. $\mathrm{kctd} 15 \mathrm{a} / \mathrm{b}$ knockdown led to measurable expansions of tfap2a mRNA expression within the IM populace, which is comprised of renal progenitors that give rise to the pronephros (Figure 6A). In WT flatmounts, the rostral end of tfap2a IM expression aligns with the somite 8 landmark. However, in $k c t d 15 a / b$ morphants, this expression domain extends in the rostral direction well beyond somite 8 . We also noted elevated tfap2a expression in kctd15a/b-deficient hindbrain regions (Figure 6A). Then, we surveyed tfap2a IM fluorescent intensity and standardized the region of data collection by tracking values from somite 7 to the end of the pre-somitic mesoderm. Plot overlay of tfap2a fluorescent intensity profiles from representative samples revealed morphant IM signal was consistently elevated above WT levels (Figure 6B). Further, tfap2a mean fluorescent intensity was significantly higher in morphants compared to WT controls (Figure 6C,D). This tfap2a transcript pulse equilibrates in the pronephros as embryonic development progresses, as normal levels of mRNA were detected in $k c t d 15 a / b$ morphants and crispants by $24 \mathrm{hpf}$ (data not shown).

Next, we sought to determine if $k c t d 15 a$ and $k c t d 15 b$ expression are affected by tfap2a-deficiency. trm mutants exhibited considerable reductions in kctd15a and kctd15b mRNA expression at 24 hpf as compared to WT controls (Figure 6E). These visual reductions in $k c t d 15 a / b$ expression were observed in both neural and pronephric tissues. Then we tested if overexpression of tfap2a via a heat-shock inducible transgene is sufficient to activate $k c t d 15 a / b$ transcription. $h s: t f a p 2 a$ animals that underwent heat-shock treatment at the 8ss displayed a global escalation of $k c t d 15 a$ and $k c t d 15 b$ expression (Figure 6F). Surprisingly, hs:tfap2a control animals that did not undergo heat-shock treatment also exhibited increased overall kctd15a and kctd15b expression, but to a lesser extent than heat-shock treated siblings (Figure 6F). We believe this is an artifact of a leaky tfap2a transgene. Nonetheless, this data illustrates that $k c t d 15 a / b$ transcript expression is extremely sensitive to tfap2a dosage. Altogether, our findings expose a previously undescribed level of genetic interaction where tfap2a regulates the 
transcription of its own repressors $k c t d 15 a$ and $k c t d 15 b$. Excitingly, these results divulged an autoregulatory feedback loop operating in the context of nephron differentiation where tfap2a controls its own abundance through the $k c t d 15 a / b$ genetic circuit.

\section{$k c t d 15 a / b$ counterbalances tfap2a to restrict DE differentiation during pronephros segmentation}

To further substantiate that $k c t d 15 a / b$ functions in nephron development by repressing Tfap2a activity, we disrupted the stoichiometry of these factors by concomitant kctd15b knockdown and induced tfap2a overexpression at the 12 ss via the heat-shock transgene. Interestingly, tfap2a overexpression produced significantly shorter $c d h 17^{+}$tubules (Figure $A, B$ ). To account for these truncated tubules in subsequent analyses, we calculated a ratio, DE length:tubule length, to statistically compare all groups. We found no significant difference in DE:tubule percentage between WT and hs:tfap2a ${ }^{+/}$controls (Figure 7A,C). The DE fraction was similarly increased in WT + kctd15b MO, hs:tfap2a $\mathrm{a}^{+-}+k c t d 15 b \mathrm{MO}$, and hs:tfap2a HS+ groups. Remarkably, pairing kctd15b knockdown with tfap2a overexpression initiated a drastic expansion of DE marker expression that was more severe than all other experimental groups. In these dual-treated animals, ectopic s/c12a $1^{+}$cells formed in both proximal and distal nephron segments (Figure 7A). These synergistic phenotypes upon concomitant knockdown of $k c t d 15 b$ and overexpression of tfap2a corroborate the interdependence of these factors during nephron segmentation. In sum, our genetic studies divulge kctd15 paralogs as essential repressors that function during nephrogenesis to stabilize the DE lineage by antagonizing the tfap2a differentiation pathway. 


\section{Discussion}

During embryonic development, Tfap2 genes are dose-dependent transcription factors, therefore require tight regulation. ${ }^{8,32-35}$ Here, our data supports a new model where kctd15 fine-tunes Tfap2a activity to control nephron segmentation. We found $k c t d 15 a / b$-deficient nephrons exhibited expanded DE and CS lineages (Figure 8). This surge in DE and CS fate occurred at the expense of neighboring segments, as PST and DL marker expression were concomitantly decreased. kctd15 loss initiated ectopic expression of DE signature genes in adjacent nephron compartments. Frequently, we detected a fraction of cells exhibiting dual segment marker expression insinuating partial fate conversion. This phenomenon of mixed segment profiles was previously documented in Abd-B Hox-deficient and tfap2a overexpressing nephrons. ${ }^{8,36}$ Previous in vitro experiments established a direct mode of interaction where KCTD15 inhibits AP-2 in whole zebrafish embryo and HEK293T lysates. ${ }^{12}$ In light of these mammalian cell line and zebrafish studies, we suspected kctd15-deficient nephron phenotypes were caused by increased Tfap2a activity. Interestingly, elevated Tfap2a protein abundance was detected in kctd15-deficient pronephroi, which diverges from previous neural crest studies. We believe accumulation of Tfap2a protein is a consequence of genetic feedback circuitry because 1) kctd15 loss expanded tfap2a mRNA expression in developing renal progenitors and 2) tfap2a promotes kctd15a and kctd15b transcription. In accordance with these observations, Tfap2a can promote its own transcription by binding to an autoregulatory element. ${ }^{37}$ If Tfap $2 a$ is unable to be repressed, positive autoregulation could explain heightened tfap2a transcript production in response to kctd15 loss-offunction.

Alternatively, it is feasible excess pronephric Tfap2a protein is present because it is not properly targeted for degradation. Previous studies have demonstrated KCTD proteins can function as adaptor molecules for E3 ubiquitin ligases to facilitate post-translational modifications of bound protein interactors. ${ }^{38}$ Further, KCTD family members are substrates for SUMOylation and ubiquitinylation, which can alter protein stability and initiate degradation respectively. There is evidence for SUMO- 
conjugation of TFAP2A, which suppresses transcriptional activation and prevents mesenchymal-toepithelial transition in breast cancer cells. ${ }^{39-42}$ Further investigation is needed to determine whether Kctd15 facilitates SUMOylation of Tfap2a in nephron precursors. Nonetheless, our $k c t d 15 a / b$-deficient genetic manipulations truncate the BTB/POZ domain and would prevent Kctd15-Tfap2a direct interactions from occurring. Because feedback circuitry is disrupted, Tfap2a activity is left unchecked in neighboring nephron segments, allowing downstream targets like DE-specific lineage factors and solute transporters to be ectopically expressed (Figure 8). In support of our model, kctd15b knockdown coupled with tfap2a overexpression yielded a synergistic phenotype where nephron cell composition was shifted toward DE assignment. In conclusion, our studies reveal a novel feedback circuit operating within the context of kidney development, where a tfap2a-kctd15 transcription factor-repressor module programs DE/TAL differentiation.

Our studies help to fill a key void in the literature by addressing developmental signaling required for $\mathrm{DE} / \mathrm{TAL}$ formation. Due to the anatomical constraints of studying mammalian nephron development in vivo, there is a limited knowledge of cell diversity and dynamics within the maturing LOH anlagen. Zebrafish studies provide a complementary launch point to explore the how functionality of candidate factors, such as tfap2a and kctd15, create nephron cell-types in vertebrates. Recently, scRNA-seq and fate-mapping in mice revealed early forming LOH (juxtamedullary) and late forming LOH (cortical) have distinct gene signatures. ${ }^{43} \mathrm{LOH}$ outgrowth from the S-shaped tubule is adaptive and oriented by longrange cues from medullary collecting ducts, however signaling pathways navigating this process have yet to be identified. ${ }^{44}$ Current efforts to advance personalized medicine by building patient-derived kidney organoids face challenges in achieving expression of $\mathrm{LOH}$-specific genes. ${ }^{45}$ Hence, there is utility in using animal models to assemble a working genetic framework that could enrich for LOH development in culture conditions. 
A compelling TFAP2A GRN candidate that warrants further investigation is KCTD1, which shares greater than $78 \%$ sequence homology with $\mathrm{KCTD} 15 .{ }^{46} \mathrm{KCTD} 1$ can directly inhibit TFAP2A as well as suppress canonical Wnt signaling, exhibiting congruence with known KCTD15 molecular mechanisms. ${ }^{47,48}$ Human KCTD1 mutations cause scalp-ear-nipple (SEN) syndrome, which involves the formation of toxic amyloid-like aggregates thought to influence disease state. ${ }^{49}$ With the advent of prenatal screening and genotyping strategies, KCTD1 lesions have been linked to renal hypoplasia. ${ }^{50}$ Along these lines, mice harboring a Kctd1 mutation die perinatally due to kidney failure associated with defective ion homeostasis. Transcriptional profiling of these mutant kidneys identified 102 significantly upregulated genes, including known TFAP2A targets. ${ }^{51}$ TFAP2A and TFAP2B null mice also manifest lethal kidney defects. ${ }^{52,53}$ Ultimately, our genetic studies elucidating TFAP2-KCTD network mechanisms in developing zebrafish nephrons can catalyze candidate identification and refine prenatal screening for renal defects. 


\section{Author contributions}

B.E.C. and R.A.W. designed the experiments. B.E.C., E.G.C., and A.G. conducted the experiments.

B.E.C., E.G.C., A.G., and R.A.W. analyzed the experiments. B.E.C. and R.A.W. wrote the manuscript.

\section{Disclosures}

The authors have nothing to disclose.

\section{References}

1. Costantini, F., \& Kopan, R. (2010). Patterning a complex organ: branching morphogenesis and nephron segmentation in kidney development. Developmental cell, 18(5), 698-712.

2. Romagnani, P., Lasagni, L., \& Remuzzi, G. (2013). Renal progenitors: an evolutionary conserved strategy for kidney regeneration. Nature Reviews Nephrology, 9(3), 137.

3. Desgrange, A., \& Cereghini, S. (2015). Nephron patterning: lessons from Xenopus, zebrafish, and mouse studies. Cells, 4(3), 483-499.

4. Wingert, R. A., Selleck, R., Yu, J., Song, H. D., Chen, Z., Song, A., .. \& Davidson, A. J. (2007). The cdx genes and retinoic acid control the positioning and segmentation of the zebrafish pronephros. PLoS genetics, 3(10), e189.

5. Wingert, R. A., \& Davidson, A. J. (2008). The zebrafish pronephros: a model to study nephron segmentation. Kidney international, 73(10), 1120-1127.

6. Poureetezadi, S. J., Cheng, C. N., Chambers, J. M., Drummond, B. E., \& Wingert, R. A. (2016). Prostaglandin signaling regulates nephron segment patterning of renal progenitors during zebrafish kidney development. Elife, 5, e17551. 
7. Chambers, J. M., Poureetezadi, S. J., Addiego, A., Lahne, M., \& Wingert, R. A. (2018). ppargc1a controls nephron segmentation during zebrafish embryonic kidney ontogeny. eLife, 7 , e40266.

8. Chambers, B. E., Gerlach, G. F., Clark, E. G., Chen, K. H., Levesque, A. E., Leshchiner, I., ... \& Wingert, R. A. (2019). Tfap2a is a novel gatekeeper of nephron differentiation during kidney development. Development, dev-172387.

9. Eckert, D., Buhl, S., Weber, S., Jäger, R., \& Schorle, H. (2005). The AP-2 family of transcription factors. Genome biology, 6(13), 246.

10. Gharbi, N., Zhao, X. F., Ellingsen, S., \& Fjose, A. (2012). Zebrafish enhancer trap line showing maternal and neural expression of kctd15a. Development, growth \& differentiation, 54(2), 241252.

11. Liu, Z., Xiang, Y., \& Sun, G. (2013). The KCTD family of proteins: structure, function, disease relevance. Cell \& bioscience, 3(1), 45 .

12. Zarelli, V. E., \& Dawid, I. B. (2013). Inhibition of neural crest formation by Kctd15 involves regulation of transcription factor AP-2. Proceedings of the National Academy of Sciences, 110(8), 2870-2875.

13. Wong, T. C., Rebbert, M., Wang, C., Chen, X., Heffer, A., Zarelli, V. E., ... Zhao, H. (2016). Genes regulated by potassium channel tetramerization domain containing $15(\mathrm{Kctd} 15)$ in the developing neural crest. The International journal of developmental biology, 60(4-6), 159-166. doi:10.1387/ijdb.160058id

14. Zarelli, V. E., \& Dawid, I. B. (2013). The BTB-containing protein Kctd15 is SUMOylated in vivo. PloS one, 8(9), e75016.

15. Gamero-Villarroel, C., González, L. M., Rodríguez-López, R., Albuquerque, D., Carrillo, J. A., García-Herráiz, A., .. \& Gervasini, G. (2017). Influence of TFAP2B and KCTD15 genetic variability on personality dimensions in anorexia and bulimia nervosa. Brain and behavior, 7(9), e00784. 
16. Smaldone, G., Pirone, L., Capolupo, A., Vitagliano, L., Monti, M. C., Di Gaetano, S., \& Pedone, E. (2018). The essential player in adipogenesis GRP78 is a novel KCTD15 interactor. International journal of biological macromolecules, 115, 469-475.

17. Williams, M. J., Almén, M. S., Fredriksson, R., \& Schiöth, H. B. (2012). What model organisms and interactomics can reveal about the genetics of human obesity. Cellular and Molecular Life Sciences, 69(22), 3819-3834.

18. Williams, M. J., Goergen, P., Rajendran, J., Zheleznyakova, G., Hägglund, M. G., Perland, E., ... \& Schiöth, H. B. (2014). Obesity-linked homologues TfAP-2 and Twz establish meal frequency in Drosophila melanogaster. PLoS genetics, 10(9), e1004499.

19. Westerfield, M. (1993). The Zebrafish Book. Eugene, USA: University of Oregon Press.

20. Kimmel, C. B., Ballard, W. W., Kimmel, S. R., Ullmann, B., \& Schilling, T. F. (1995). Stages of embryonic development of the zebrafish. Developmental dynamics, 203(3), 253-310.

21. Marra, A. N., Chambers, B. E., Chambers, J. M., Drummond, B. E., Adeeb, B. D., Wesselman, H. M., ... \& Wingert, R. A. (2019). Visualizing gene expression during zebrafish pronephros development and regeneration. Methods in cell biology, 154, 183-215.

22. Gagnon, J. A., Valen, E., Thyme, S. B., Huang, P., Ahkmetova, L., Pauli, A., ... \& Schier, A. F. (2014). Efficient mutagenesis by Cas9 protein-mediated oligonucleotide insertion and largescale assessment of single-guide RNAs. PloS one, 9(5), e98186.

23. Bhat, N., Kwon, H. J., \& Riley, B. B. (2013). A gene network that coordinates preplacodal competence and neural crest specification in zebrafish. Developmental biology, 373(1), $107-$ 117.

24. Kantarci, H., Edlund, R. K., Groves, A. K., \& Riley, B. B. (2015). Tfap2a promotes specification and maturation of neurons in the inner ear through modulation of Bmp, Fgf and notch signaling. PLoS genetics, 11(3), e1005037.

25. Dutta, S., \& Dawid, I. B. (2010). Kctd15 inhibits neural crest formation by attenuating Wnt/ $\beta$ catenin signaling output. Development, 137(18), 3013-3018. 
26. Takahashi, C., Suzuki, T., Nishida, E., \& Kusakabe, M. (2012). Identification and characterization of Xenopus kctd15, an ectodermal gene repressed by the FGF pathway. International Journal of Developmental Biology, 56(5), 393-402.

27. Harding, S. D., Armit, C., Armstrong, J., Brennan, J., Cheng, Y., Haggarty, B., ... \& Sharghi, M. (2011). The GUDMAP database-an online resource for genitourinary research. Development, 138(13), 2845-2853.

28. McMahon, A. P., Aronow, B. J., Davidson, D. R., Davies, J. A., Gaido, K. W., Grimmond, S., ... \& Zhang, P. (2008). GUDMAP: the genitourinary developmental molecular anatomy project. Journal of the American Society of Nephrology, 19(4), 667-671.

29. Heffer, A., Marquart, G. D., Aquilina-Beck, A., Saleem, N., Burgess, H. A., \& Dawid, I. B. (2017). Generation and characterization of Kctd15 mutations in zebrafish. PloS one, 12(12), e0189162.

30. Cheng, C. N., \& Wingert, R. A. (2015). Nephron proximal tubule patterning and corpuscles of Stannius formation are regulated by the sim1a transcription factor and retinoic acid in zebrafish. Developmental biology, 399(1), 100-116.

31. Naylor, R. W., Chang, H. H. G., Qubisi, S., \& Davidson, A. J. (2018). A novel mechanism of gland formation in zebrafish involving transdifferentiation of renal epithelial cells and live cell extrusion. Elife, 7, e38911.

32. Dooley, C. M., Wali, N., Sealy, I. M., White, R. J., Stemple, D. L., Collins, J. E., \& BuschNentwich, E. M. (2019). The gene regulatory basis of genetic compensation during neural crest induction. PLoS genetics, 15(6), e1008213.

33. Hammer, S., Toenjes, M., Lange, M., Fischer, J. J., Dunkel, I., Mebus, S., ... \& Sperling, S. (2008). Characterization of TBX20 in human hearts and its regulation by TFAP2. Journal of cellular biochemistry, 104(3), 1022-1033.

34. Van Otterloo, E., Li, H., Jones, K. L., \& Williams, T. (2018). AP-2 $\alpha$ and AP-2 $\beta$ cooperatively orchestrate homeobox gene expression during branchial arch patterning. Development, 145(2), dev157438. 
35. Erickson, P. A., Baek, J., Hart, J. C., Cleves, P. A., \& Miller, C. T. (2018). Genetic dissection of a supergene implicates Tfap2a in craniofacial evolution of threespine sticklebacks. Genetics, 209(2), 591-605.

36. Magella, B., Mahoney, R., Adam, M., \& Potter, S. S. (2018). Reduced Abd-B Hox function during kidney development results in lineage infidelity. Developmental biology, 438(2), 84-93.

37. Imhof, A., Schuierer, M., Werner, O., Moser, M., Roth, C., Bauer, R., \& Buettner, R. (1999). Transcriptional regulation of the AP-2 $\alpha$ promoter by BTEB-1 and AP-2rep, a novel wt-1/egrrelated zinc finger repressor. Molecular and Cellular Biology, 19(1), 194-204.

38. Pinkas, D. M., Sanvitale, C. E., Bufton, J. C., Sorrell, F. J., Solcan, N., Chalk, R., ... \& Bullock, A. N. (2017). Structural complexity in the KCTD family of Cullin3-dependent E3 ubiquitin ligases. Biochemical Journal, 474(22), 3747-3761.

39. Eloranta, J. J., \& Hurst, H. C. (2002). Transcription factor AP-2 interacts with the SUMOconjugating enzyme UBC9 and is sumolated in vivo. Journal of Biological Chemistry, 277(34), 30798-30804.

40. Berlato, C., Chan, K. V., Price, A. M., Canosa, M., Scibetta, A. G., \& Hurst, H. C. (2011). Alternative TFAP2A isoforms have distinct activities in breast cancer. Breast Cancer Research, 13(2), R23.

41. Bogachek, M. V., Chen, Y., Kulak, M. V., Woodfield, G. W., Cyr, A. R., Park, J. M., ... \& Weigel, R. J. (2014). Sumoylation pathway is required to maintain the basal breast cancer subtype. Cancer cell, 25(6), 748-761.

42. Bogachek, Maria V., James P. De Andrade, and Ronald J. Weigel. "Regulation of epithelialmesenchymal transition through SUMOylation of transcription factors." Cancer research 75.1 (2015): 11-15.

43. Ransick, A., Lindström, N. O., Liu, J., Qin, Z., Guo, J. J., Alvarado, G. F., .. \& McMahon, A. P. (2019). Single Cell Profiling Reveals Sex, Lineage and Regional Diversity in the Mouse Kidney. bioRxiv, 673335. 
44. Chang, C. H., \& Davies, J. A. (2019). In developing mouse kidneys, orientation of loop of Henle growth is adaptive and guided by long-range cues from medullary collecting ducts. Journal of anatomy.

45. Schutgens, F., Rookmaaker, M. B., Margaritis, T., Rios, A., Ammerlaan, C., Jansen, J., ... \& Yengej, F. Y. (2019). Tubuloids derived from human adult kidney and urine for personalized disease modeling. Nature biotechnology, 37(3), 303.

46. Pirone, L., Smaldone, G., Spinelli, R., Barberisi, M., Beguinot, F., Vitagliano, L., ... \& Pedone, E. (2019). KCTD1: A novel modulator of adipogenesis through the interaction with the transcription factor AP2a. Biochimica et Biophysica Acta (BBA)-Molecular and Cell Biology of Lipids, 1864(12), 158514.

47. Ding, X., Luo, C., Zhou, J., Zhong, Y., Hu, X., Zhou, F., ... \& Gao, X. (2009). The interaction of KCTD1 with transcription factor AP-2 $\alpha$ inhibits its transactivation. Journal of cellular biochemistry, 106(2), 285-295.

48. Li, X., Chen, C., Wang, F., Huang, W., Liang, Z., Xiao, Y., ... \& Ding, X. (2014). KCTD1 suppresses canonical Wnt signaling pathway by enhancing $\beta$-catenin degradation. PloS one, 9(4), e94343.

49. Smaldone, G., Balasco, N., Pirone, L., Caruso, D., Di Gaetano, S., Pedone, E. M., \& Vitagliano, L. (2019). Molecular basis of the scalp-ear-nipple syndrome unraveled by the characterization of disease-causing KCTD1 mutants. Scientific reports, 9(1), 10519.

50. Gray, K. J., Wilkins-Haug, L. E., Herrig, N. J., \& Vora, N. L. (2019). Fetal phenotypes emerge as genetic technologies become robust. Prenatal diagnosis, 39(9), 811-817.

51. Kumar, S., Rathkolb, B., Sabrautzki, S., Krebs, S., Kemter, E., Becker, L., ... \& Garrett, L. (2017). Standardized, systemic phenotypic analysis reveals kidney dysfunction as main alteration of Kctd1 I27N mutant mice. Journal of biomedical science, 24(1), 57. 
52. Zhang, J., Hagopian-Donaldson, S., Serbedzija, G., Elsemore, J., Plehn-Dujowich, D., McMahon, A. P., ... \& Williams, T. (1996). Neural tube, skeletal and body wall defects in mice lacking transcription factor AP-2. Nature, 381(6579), 238.

53. Moser, M., Pscherer, A., Roth, C., Becker, J., Mücher, G., Zerres, K., .. \& Fässler, R. (1997). Enhanced apoptotic cell death of renal epithelial cells in mice lacking transcription factor AP-2 $\beta$. Genes \& development, 11(15), 1938-1948. 


\section{Figure Legends}

Fig. 1 | $k c t d 15 a$ and $k c t d 15 b$ are expressed in developing distal nephron precursors. (A) WISH of kctd15a and kctd15b during WT embryogenesis. Black arrowheads mark expression within developing renal field. Scale bar: $200 \mu \mathrm{m}$. (B) WISH of kctd15a and kctd15b. Black arrowheads mark expression within distal nephron precursors. Scale bar: $200 \mu \mathrm{m}$. (C) FISH of kctd15a (magenta) and tfap2a (green) in 12 ss WT flatmount. Grey box indicates featured region in right panel. Grey dots demarcate renal progenitor stripe. Scale bars: $100 \mu \mathrm{m}$ (left), $5 \mu \mathrm{m}$ (right). (D) FISH of tfap2a (green) and kctd15a (magenta) at $24 \mathrm{hpf}$. Grey box represents region imaged at higher magnification (bottom panels). Cyan encircles an example of a co-expressing cell. Scale bars: $35 \mu \mathrm{m}$ (top), $5 \mu \mathrm{m}$ (bottom). (E) FISH of tfap2a (green) and kctd15b (magenta) at $24 \mathrm{hpf}$. Grey box represents region imaged at higher magnification (bottom panels). Cyan encircles an example of a co-expressing cell. Scale bars: $35 \mu \mathrm{m}$ (top), $5 \mu \mathrm{m}$ (bottom).

Fig. 2 | kctd15a/b loss-of-function initiates expansion of DE lineage markers. (A) WISH of kcnj1a.1 (purple) at 24 hpf in WT, WT + kctd15a cRNA, kctd15 MO, and kctd15 crispants. Scale bar: 35 $\mu \mathrm{m}$. (B) Absolute length quantification of kcnj1a.1. Black bracket clusters groups together for collective comparison. (C) WISH of slc12a1 (purple) and slc12a3 (red) at 24 hpf in WT, WT + kctd15a cRNA, kctd15 MO, and kctd15 crispants. Scale bar: $35 \mu \mathrm{m}$. (D) Absolute length quantification of slc12a1. Black bracket clusters groups together for collective comparison. (E) IF of Slc12a1/2 (magenta) and laminin (green) in WT and kctd15a/b MO at $24 \mathrm{hpf}$. White arrowheads mark limits of Slc12a1/2 pronephric expression. Scale bar: $35 \mu \mathrm{m}$. (F) Fluorescent intensity plot of 3 WT (greyscale) and 3 kctd15a/b MO individuals (blue). Green dashed line equates to WT Slc12a1/2 intensity threshold (au). Red dashed line represents WT maximum Slc12a1/2 intensity value (au). (G) Mean Slc12a1/2 fluorescent intensity graph. (H) Violin plot displaying point distribution of Slc12a1/2 intensity values collected. (I) Quantification of number of Slc12a1 ${ }^{+}$pronephric cells. $\mathrm{n} \geq 3 . \quad{ }^{*} \mathrm{P}<0.05$; ${ }^{* *} \mathrm{P}<0.01$; 
${ }^{* * *} \mathrm{P}<0.001$. Data are mean \pm s.d. Absolute lengths compared by ANOVA. Mean fluorescent intensity and cell counts analyzed by unpaired t-tests.

Fig. 3 | kctd15a/b-deficiency elevates CS differentiation. (A) WISH of slc12a1 (red) and stc1 (purple) at $24 \mathrm{hpf}$ in WT, kctd15 MO, and kctd15 crispants. Black arrowheads indicate proximal stray stc1+ cells clearly separate from the main CS cluster in kctd15a/b MO. Scale bar: $35 \mu \mathrm{m}$. (B) Quantification of $s t c 1^{+}$cell number per nephron. Black bracket clusters groups together for collective comparison. (C) IF of laminin (green) in $24 \mathrm{hpf} \mathrm{WT} \mathrm{and} \mathrm{kctd15a/b} \mathrm{MO.} \mathrm{Premature} \mathrm{basement} \mathrm{membrane}$ formation occurs in $k c t d 15 a / b$ morphant, which separates the budding CS cell cluster (white dotted circle) from the pronephric tubule. Scale bar: $5 \mu \mathrm{m} . \mathrm{n} \geq 3$. ${ }^{*} \mathrm{P}<0.05$; ${ }^{* *} \mathrm{P}<0.01$; ${ }^{* \star *} \mathrm{P}<0.001$. Data are mean \pm s.d. Cell counts compared by ANOVA.

Fig. 4 | Loss of $k c t d 15 a / b$ sways neighboring pronephric fates to express DE signature. (A) FISH of kcnj1a.1 (green) and slc12a3 (red) at $24 \mathrm{hpf}$ in WT and kctd15a/b MO. Cyan arrowheads denote kcnj1a.1 boundaries. Grey box indicates area featured at higher magnification in panel below. White circles represent individual cells dually expressing kcnj1a.1 and s/c12a3. Scale bars: $70 \mu \mathrm{m}$ (top), $5 \mu \mathrm{m}$ (bottom). (B) WT fluorescent intensity plot of kcnj1a.1 (green) and slc12a3 (red). (C) kctd15a/b MO fluorescent intensity plot of kcnj1a.1 (green) slc12a3 (red). White arrows indicate ectopic kcnj1a.1 signal invading the slc12a3 domain. (D) Absolute slc12a3 length quantification. (E) FISH of trpm7 (red) and kcnj1a.1 (green) at $24 \mathrm{hpf}$ in WT and kctd15a/b MO. Cyan arrowheads denote kcnj1a.1 boundaries. Grey box indicates area featured at higher magnification in below panel. White circles represent individual cells dually expressing kcnj1a.1 and trpm7. Scale bars: $70 \mu \mathrm{m}$ (top), $5 \mu \mathrm{m}$ (bottom). (F) WT fluorescent intensity plot of trpm7 (red) and kcnj1a.1 (green). (G) kctd15a/b MO fluorescent intensity plot of trpm7 (red) and kcnj1a.1 (green). White arrows indicate ectopic kcnj1a.1 signal invading the trpm7 domain. (H) Absolute trpm7 length quantification. $n \geq 3$. ${ }^{*} \mathrm{P}<0.05 ;{ }^{* *} \mathrm{P}<0.01$. Data are mean \pm s.d. Absolute lengths compared by unpaired t-tests. 
Fig. 5 | kctd15a/b knockdown expands Tfap2a protein expression in the pronephros (A) Wholemount IF of Tfap2a and Slc12a1/2 in WT and kctd15a/b MO. Scale bars: $35 \mu \mathrm{m}$ (top), $5 \mu \mathrm{m}$ (bottom). White arrowheads indicate limits of pronephric Tfap2a expression domain. Grey box highlights region at higher magnification below. Grey dotted lines demarcate pronephric tubule. (B) Fluorescent intensity plot of Tfap2a featuring one representative WT and kctd15a/b MO sample. Green arrows label differential Tfap2a signal peaks. Green bar spans region of differential expression corresponding to DE locale $(100-220 \mu \mathrm{m})$. (C) Mean fluorescent intensity (au) graph of Tfap2a across the 100-220 $\mu \mathrm{m}$ region. (D) Violin plot displaying point distribution of Tfap2a intensity values collected across the 100$220 \mu \mathrm{m}$ region. (E) Quantification of number of Tfap2a ${ }^{+}$pronephric cells. $n \geq 3$. ${ }^{* *} P<0.001$. Data are mean \pm s.d. Mean fluorescent intensity and cell counts analyzed by unpaired t-tests.

Fig. 6 | kctd15a/b and tfap2a participate in genetic crosstalk. (A) Whole-mount FISH of tfap2a (green) and smyhc1 (magenta) in 10 ss WT and kctd15a/b MO flatmounts. Yellow box indicates differential tfap2a hindbrain expression. Red arrows demarcate tfap2a IM stripe expression limits. Numbers label somite 7 and 8 landmarks. White box indicates region depicted in below panel at higher magnification. Scale bars $=100 \mu \mathrm{m}$ (above), $5 \mu \mathrm{m}$ (below). (B) Fluorescent intensity plot of tfap2a IM expression featuring one representative WT (greyscale) and kctd15a/b MO (green) sample. Blue dotted line signifies WT mean fluorescent intensity threshold. (C) Overall mean fluorescent intensity (au) graph of tfap2a IM expression. Fluorescent intensity values were collected from somite 7 to the end of the pre-somitic mesoderm. (D) Violin plot displaying point distribution of tfap2a intensity values. (E) WISH of $k c t d 15 a$ or $k c t d 15 b$ (purple) in WT and $t r m^{-/}$mutants. Inset features neural expression. Right panel features pronephric expression. Scale bars: $70 \mu \mathrm{m}$ (left), $35 \mu \mathrm{m}$ (right). (F) WISH of $k c t d 15 a$ or $k c t d 15 b$ (purple) in WT and hs:tfap2a. HS+ (red) signifies heat-shock treatment at the 8ss. Scale bar: $70 \mu \mathrm{m}$. $\mathrm{n} \geq 3$. ${ }^{* * *} \mathrm{P}<0.001$. Data are mean \pm s.d. Mean fluorescent intensity analyzed by unpaired t-tests. 
Fig. 7 | kctd15a/b-tfap2a autoregulatory feedback loop balances DE pronephric differentiation. (A) WISH of slc12a1 (DE, purple) and cdh17 (tubule, red) in hs:tfap2a transgenic background in combination with $k c t d 15 b \mathrm{MO}$ treatment. HS+ indicates application of heat-shock treatment at the $12 \mathrm{ss}$. Blue arrowheads annotate proximal and distal edges of slc12a1 expression. Scale bar: $35 \mu \mathrm{m}$. (B) Quantification of cdh17 length in control and treatment groups. (C) Bar graph depicting percent DE occupancy of the tubule. HS- = no heat-shock treatment, HS+ = heat-shock treatment. $n \geq 6 .{ }^{*} \mathrm{P}<0.05$; ${ }^{* * *} P<0.001$. Data are mean \pm s.d. Absolute lengths and percentages compared by ANOVA.

Fig. 8 | Working model illustrating the proposed role of the Kctd15-Tfap2a interaction in maturing nephron cells. WT (left), kctd15a/b-deficient context (right) at $24 \mathrm{hpf}$. Distal early (DE, cyan), corpuscle of Stannius (CS, orange), neighboring cell populations (grey). Black dashed box indicates featured distal nephron zone. Cyan arrows signify expansion of DE differentiation in $k c t d 15 a / b$-deficient nephron. Magenta circle outlines single cell featured in bottom panel. In WT, Tfap2a promotes kctd15 expression at the transcriptional level and Kctd15 protein (purple) represses Tfap2a (green) activity, allowing for proper nephron segment differentiation. In kctd15-deficiency, predicted truncated Kctd15 protein is not able to inhibit Tfap2a activity in neighboring cell populations, allowing for ectopic activation of DE lineage factors and solute transporters (s/c12a1, kcnj1a.1). 
bioRxiv preprint doi: https://doi.org/10.1101/2020.01.17.910760; this version posted January 18, 2020. The copyright holder for this preprint (which was not certified by peer review) is the author/funder, who has granted bioRxiv a license to display the preprint in perpetuity. It is made Figure 1

A

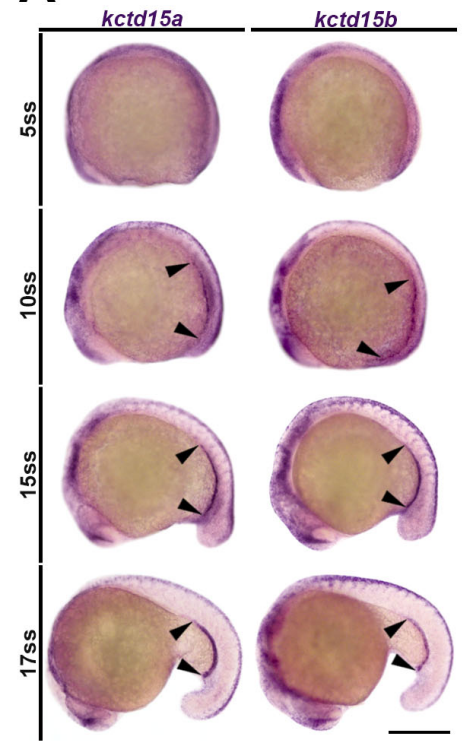

D
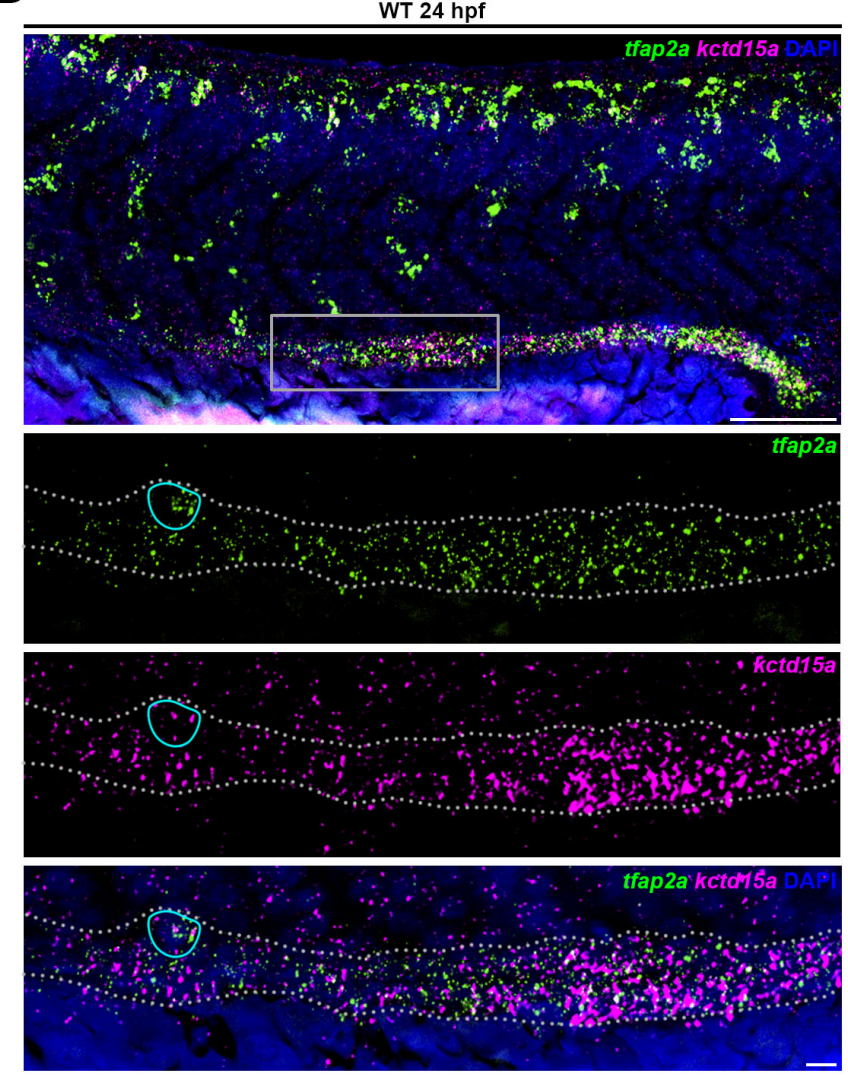

B

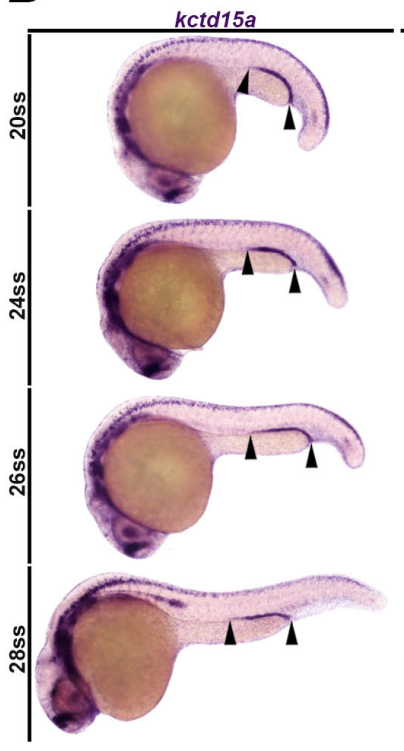

E
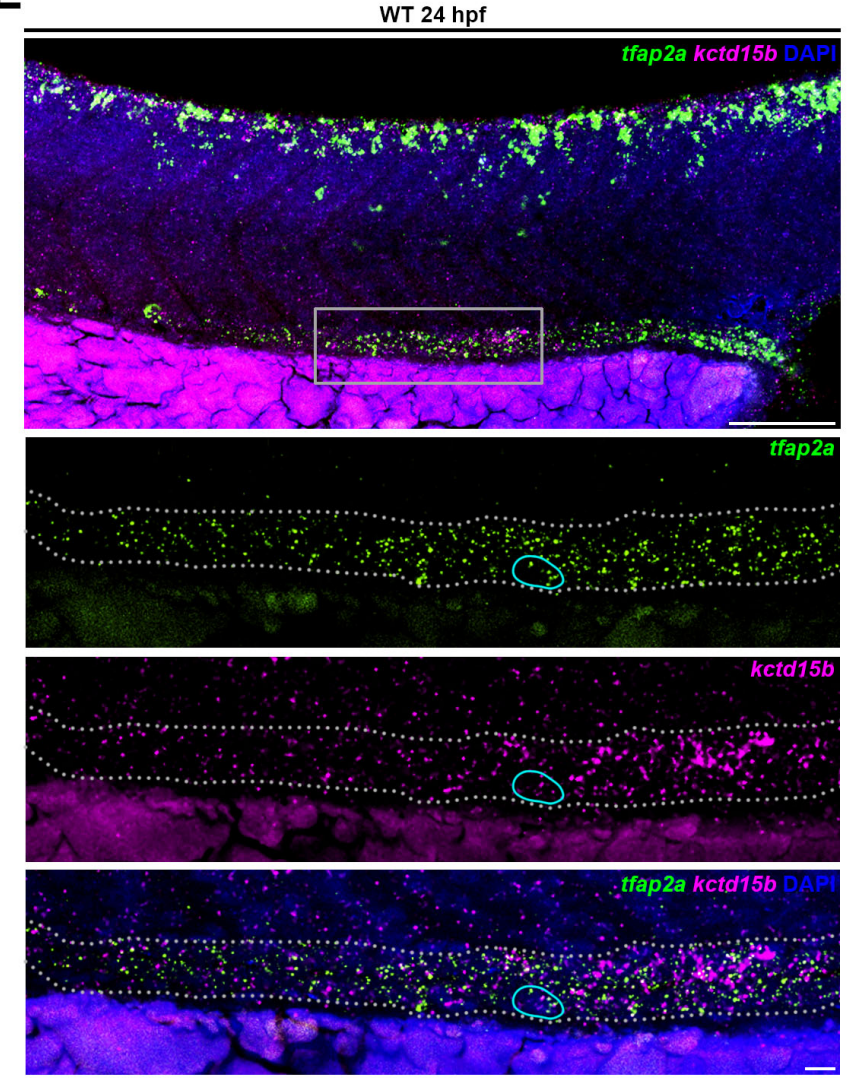
bioRxiv preprint doi: https://doi.org/10.1101/2020.01.17.910760; this version posted January 18,2020 . The copyright holder for this preprint (which was not certified by peer review) is the author/funder, who has granted bioRxiv a license to display the preprint in perpetuity. It is made

Figure 2
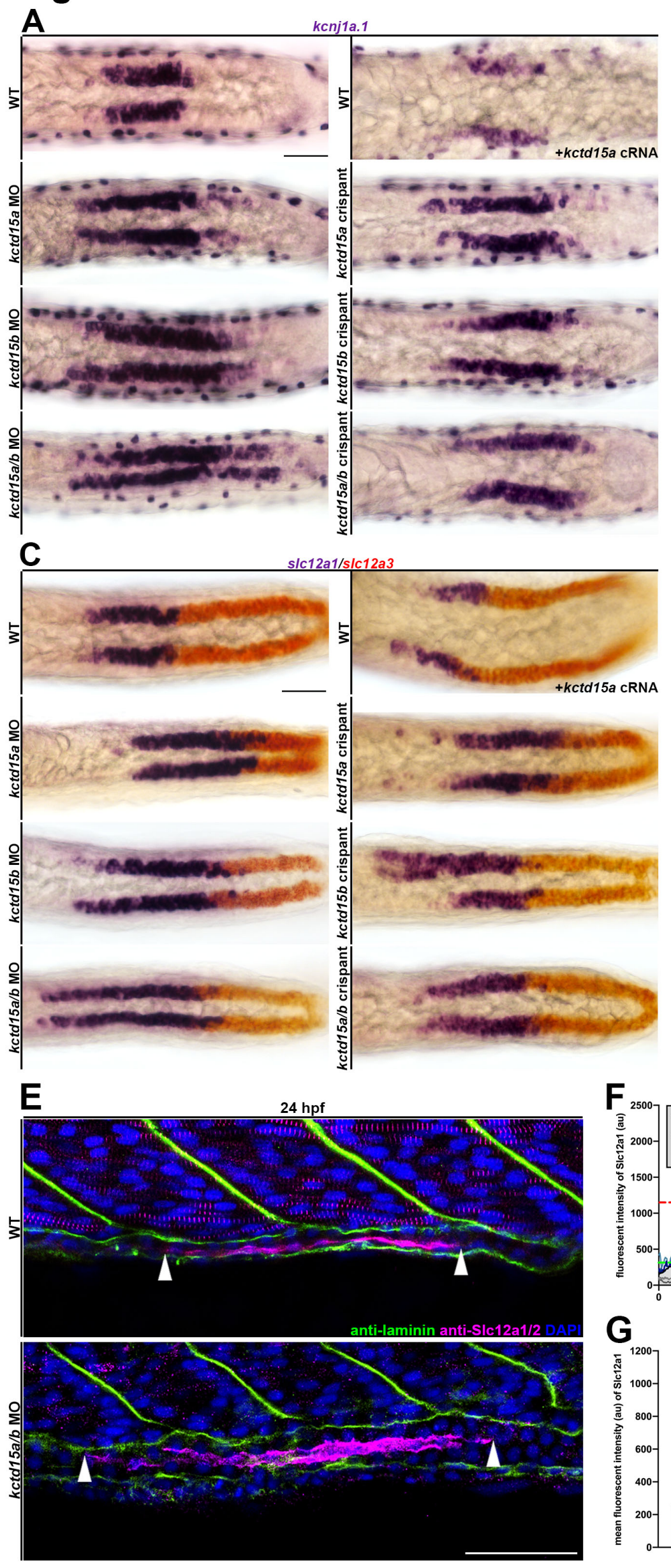
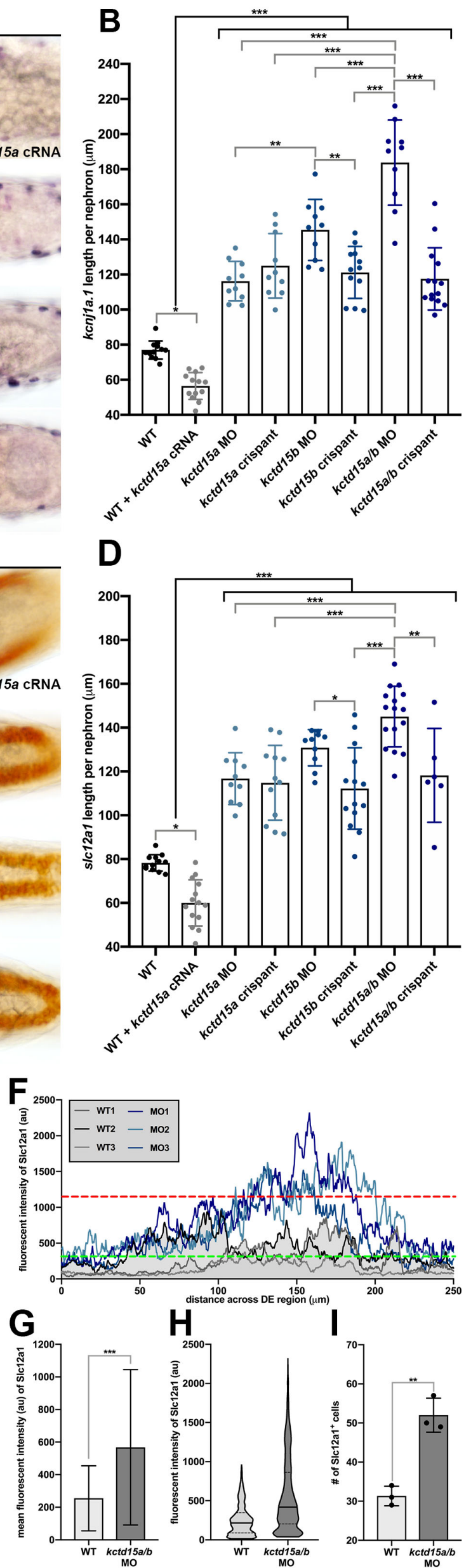
bioRxiv preprint doi: https://doi.org/10.1101/2020.01.17.910760; this version posted January 18,2020 . The copyright holder for this preprint (which was not certified by peer review) is the author/funder, who has granted bioRxiv a license to display the preprint in perpetuity. It is made Figure 3
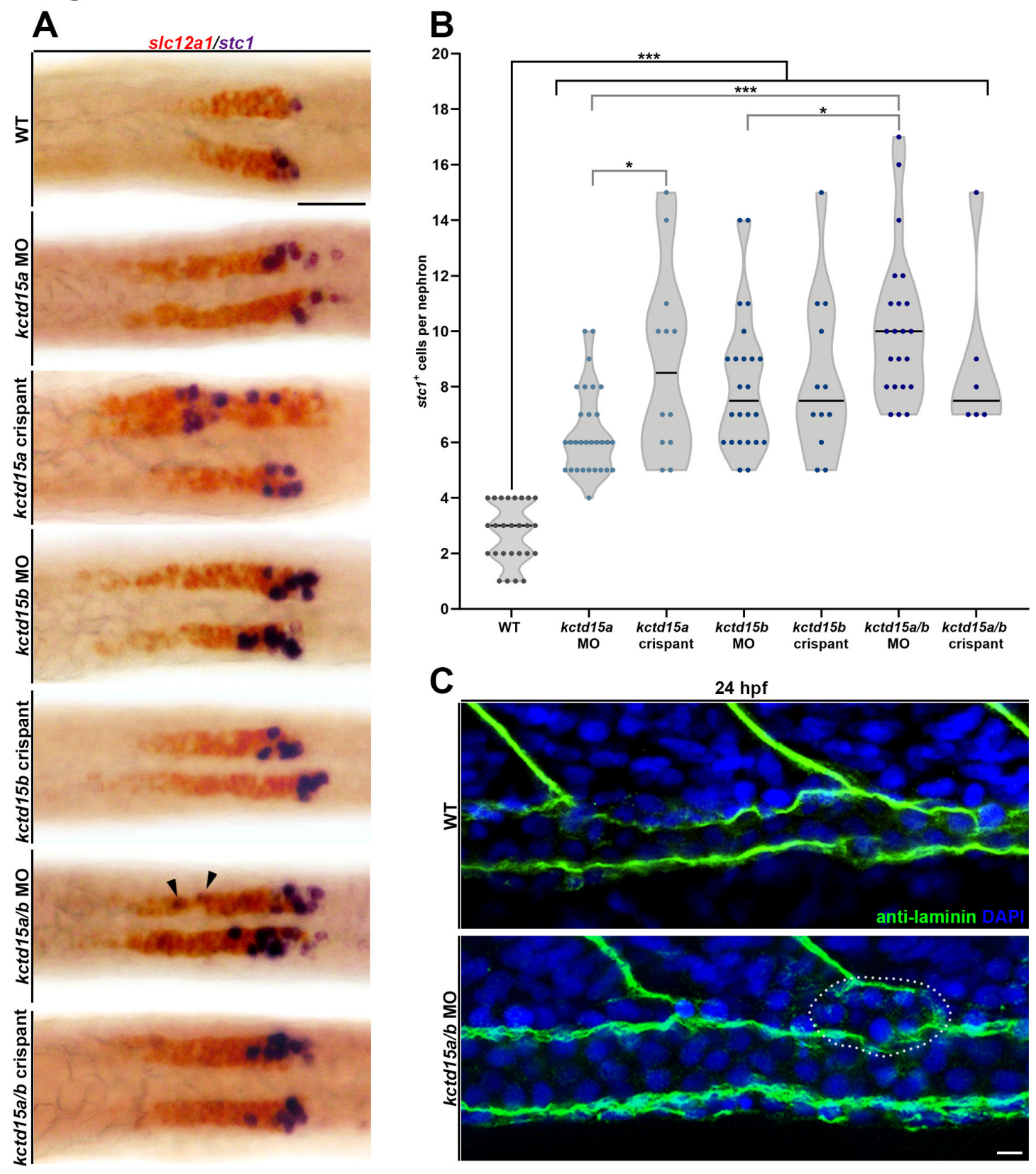
bioRxiv preprint doi: https://doi.org/10.1101/2020.01.17.910760; this version posted January 18, 2020. The copyright holder for this preprint (which was not certified by peer review) is the author/funder, who has granted bioRxiv a license to display the preprint in perpetuity. It is made Figure 4

A

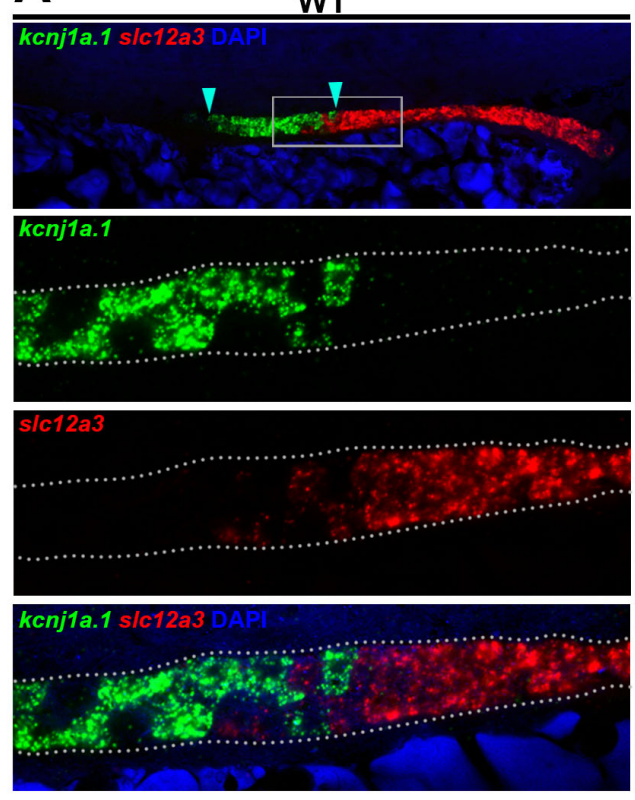

E

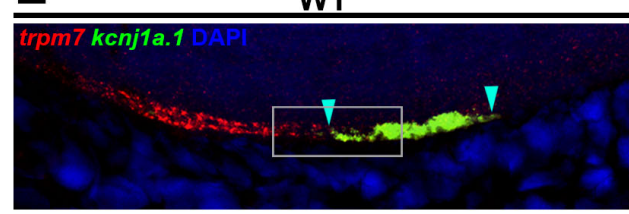

kcnj1a.1
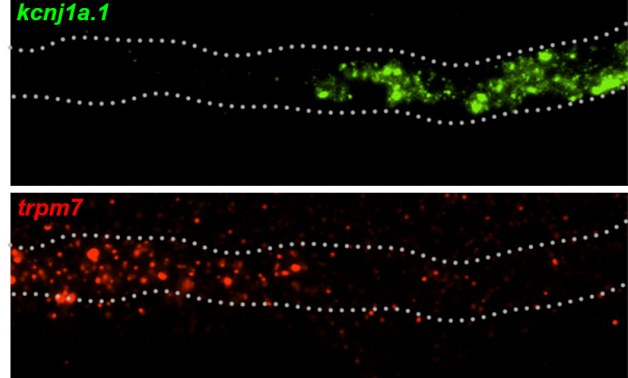

trom7 kcnj1a.1

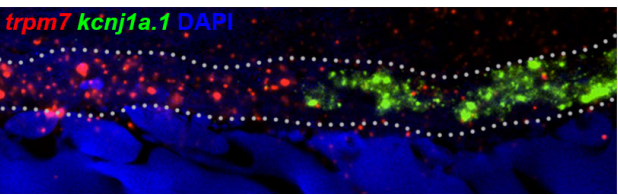

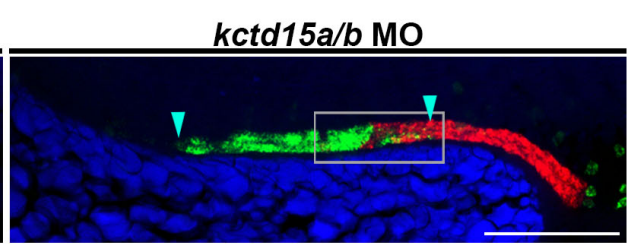
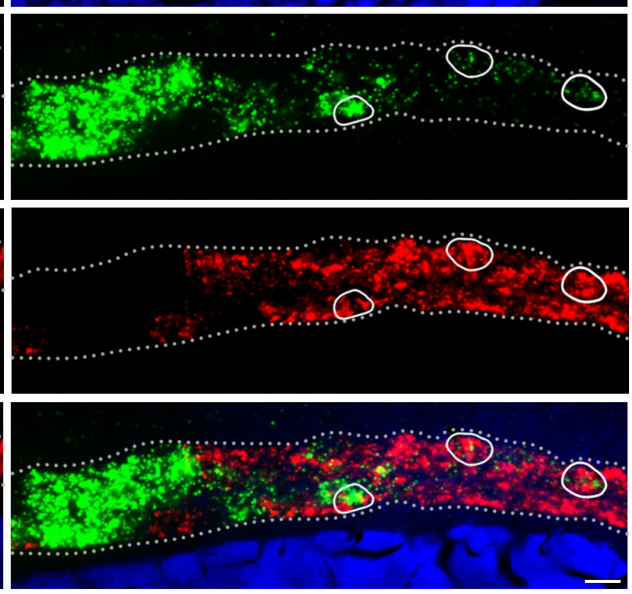

$k c t d 15 a / b$ MO
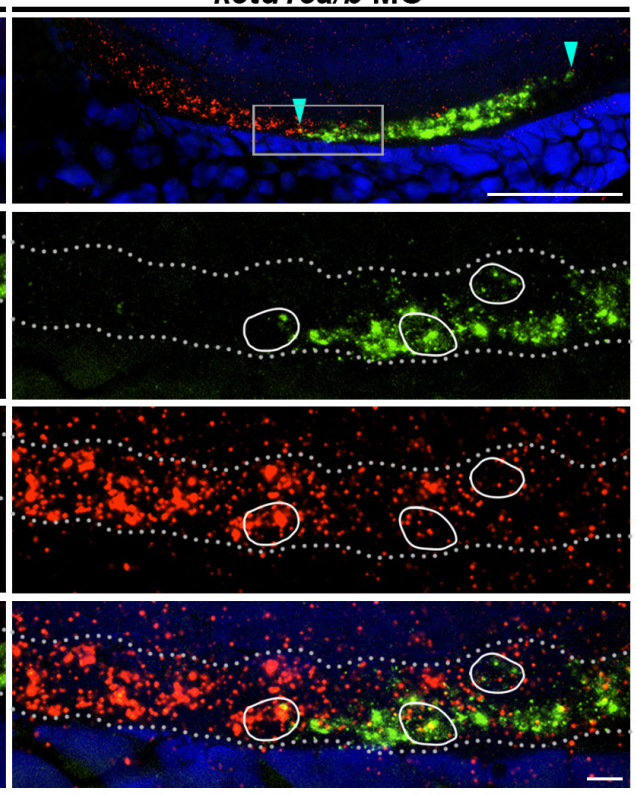

B

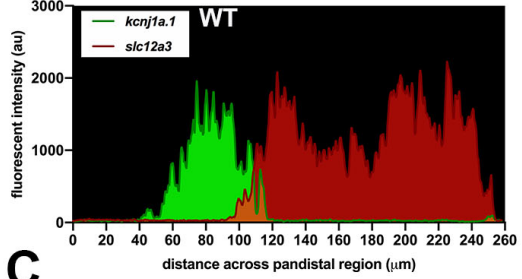

$\mathrm{C}_{3000 \quad \text { dis }}$
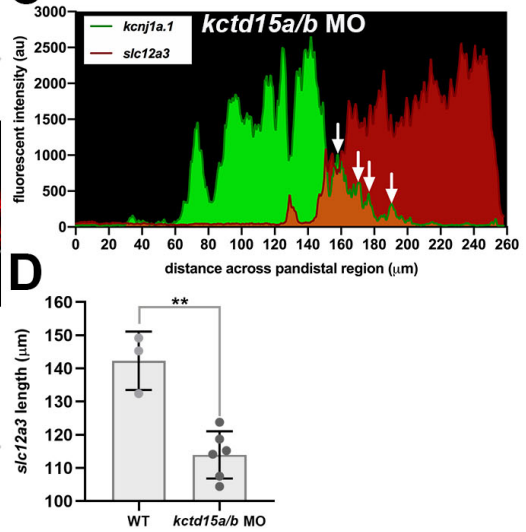

$\mathbf{F}_{250}$
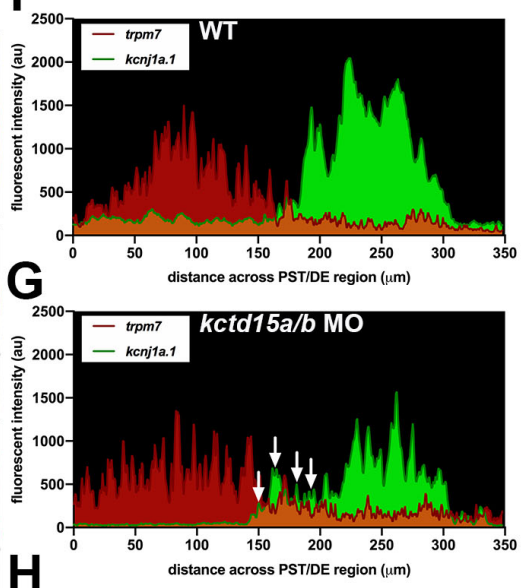

$\mathbf{H}$

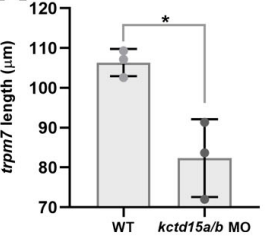


bioRxiv preprint doi: https://doi.org/10.1101/2020.01.17.910760; this version posted January 18, 2020. The copyright holder for this preprint (which was not certified by peer review) is the author/funder, who has granted bioRxiv a license to display the preprint in perpetuity. It is made

\section{Figure 5}

A
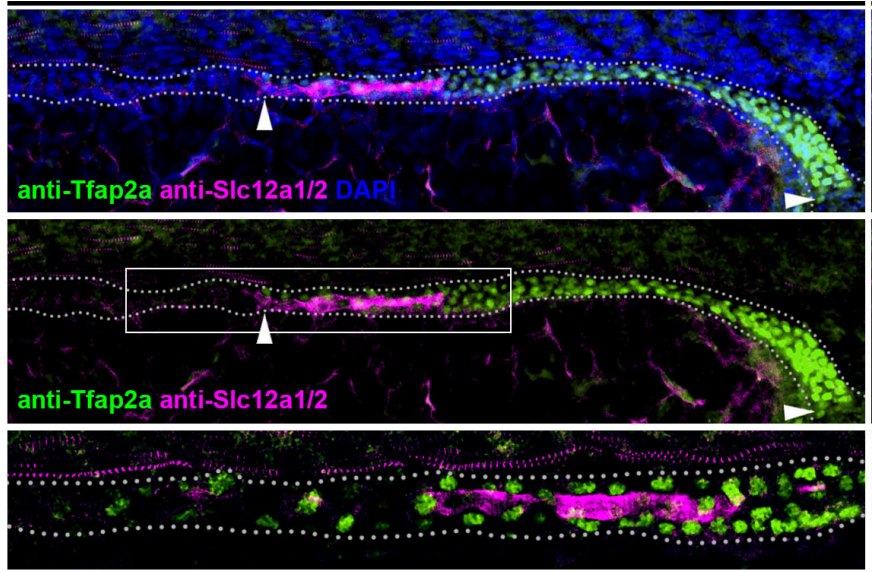

B

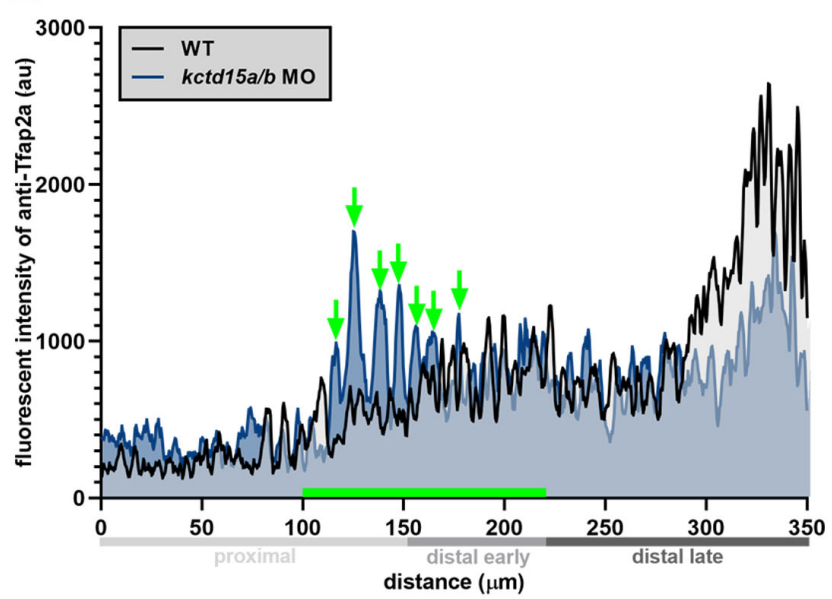

kctd15a/b MO
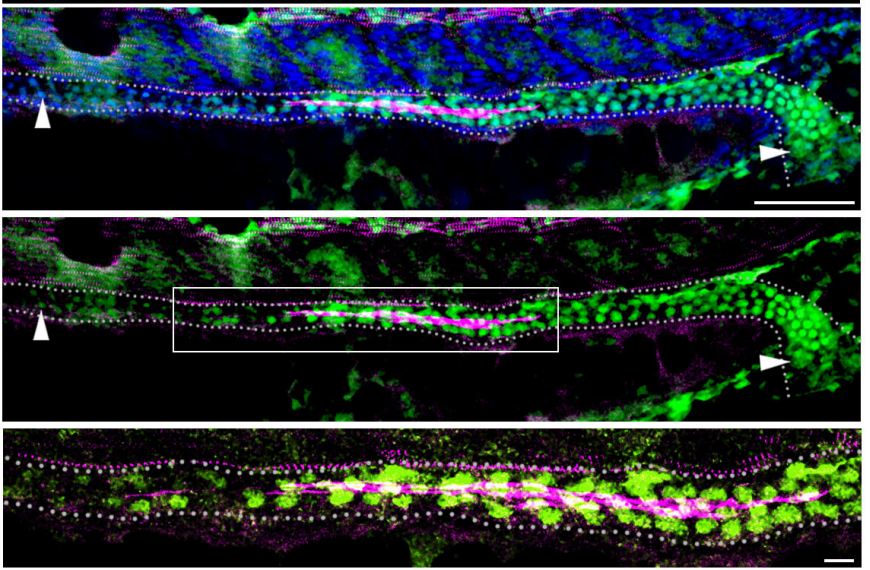

C

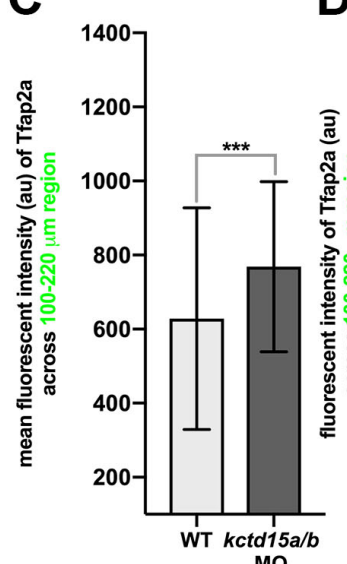

D

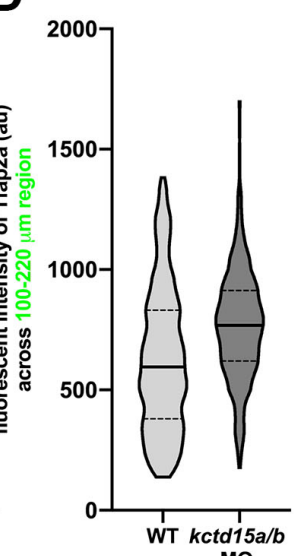

E

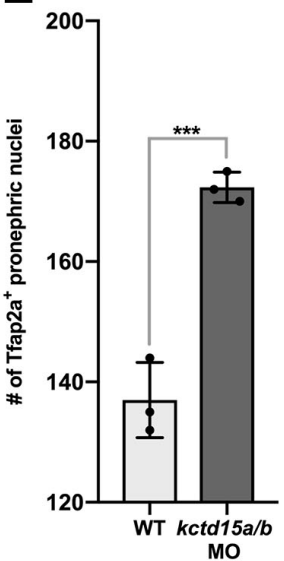
MO 
bioRxiv preprint doi: https://doi.org/10.1101/2020.01.17.910760; this version posted January 18, 2020. The copyright holder for this preprint (which was not certified by peer review) is the author/funder, who has granted bioRxiv a license to display the preprint in perpetuity. It is made

\section{Figure 6}
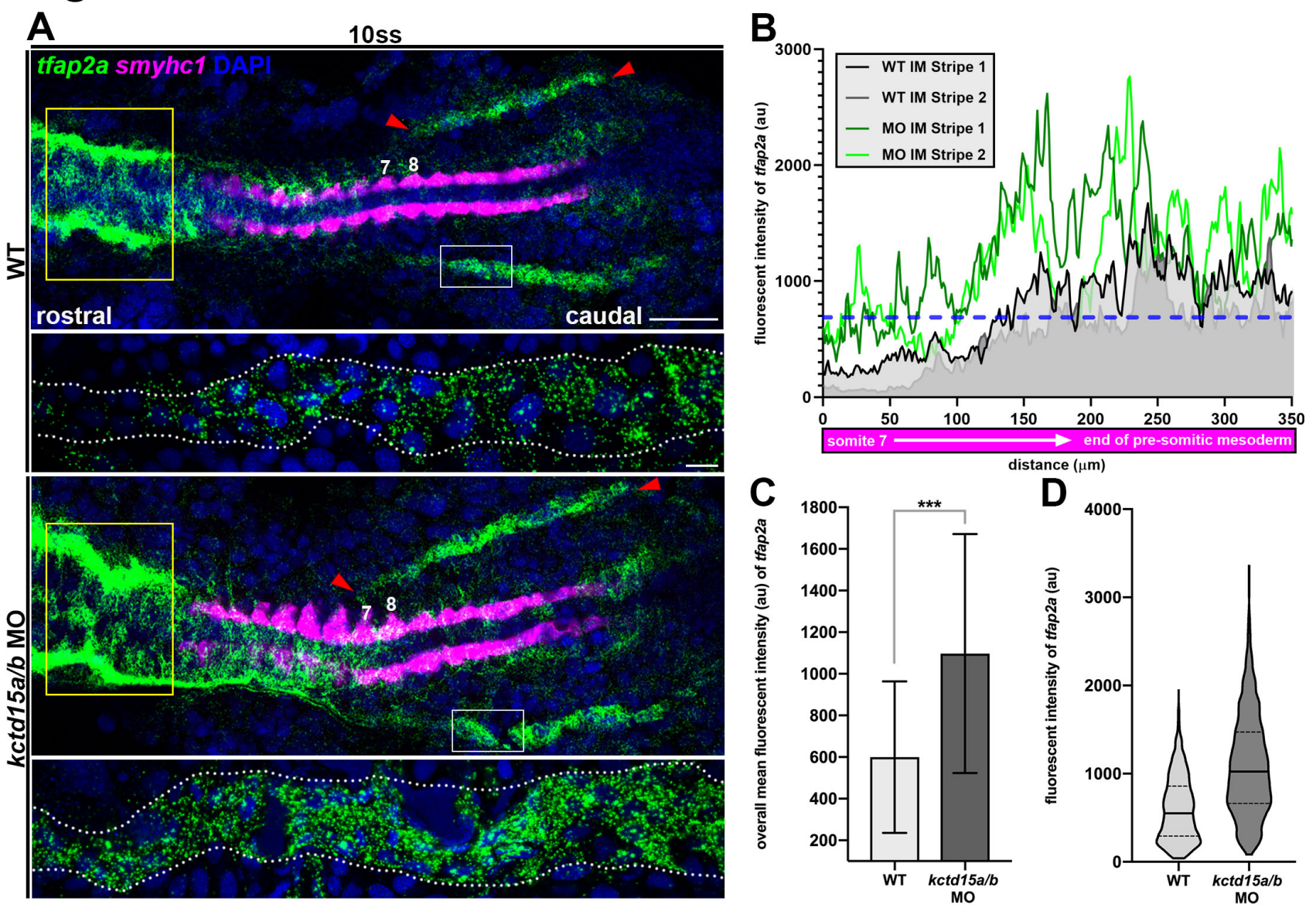

E
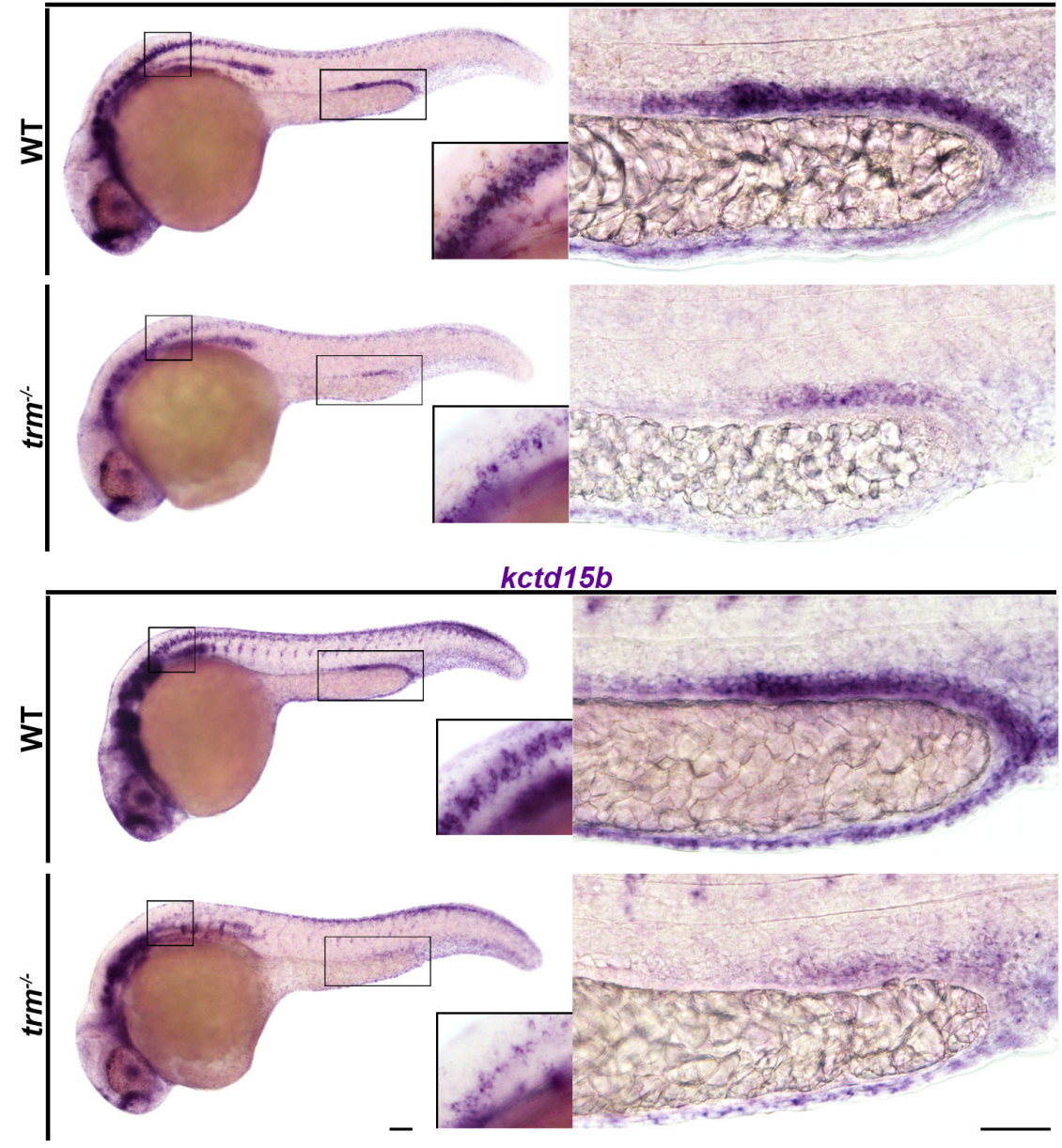

$\mathbf{F}$
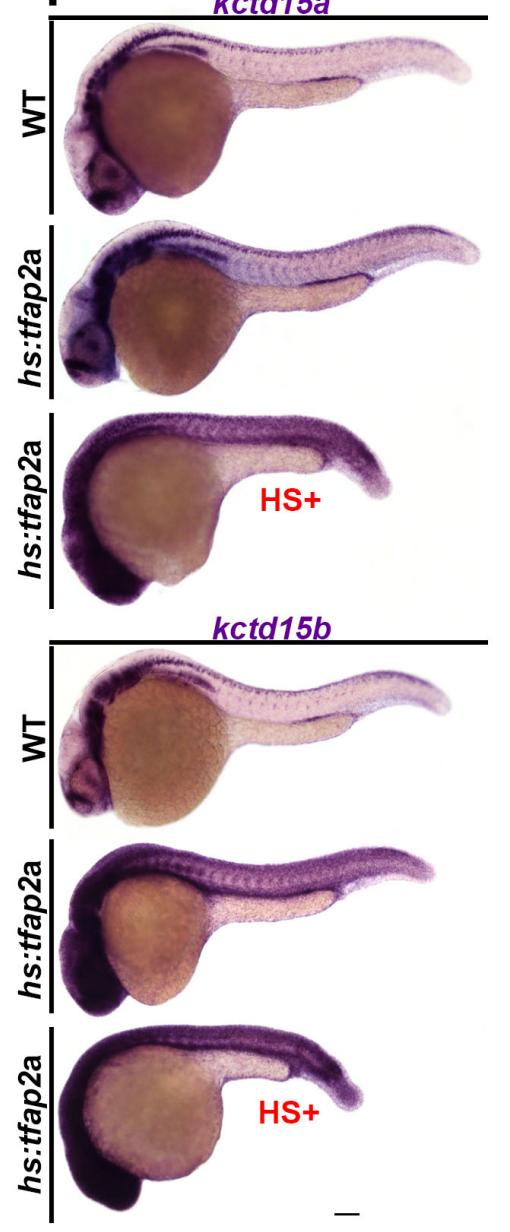
bioRxiv preprint doi: https://doi.org/10.1101/2020.01.17.910760; this version posted January 18,2020 . The copyright holder for this preprint (which was not certified by peer review) is the author/funder, who has granted bioRxiv a license to display the preprint in perpetuity. It is made

\section{Figure 7}

A

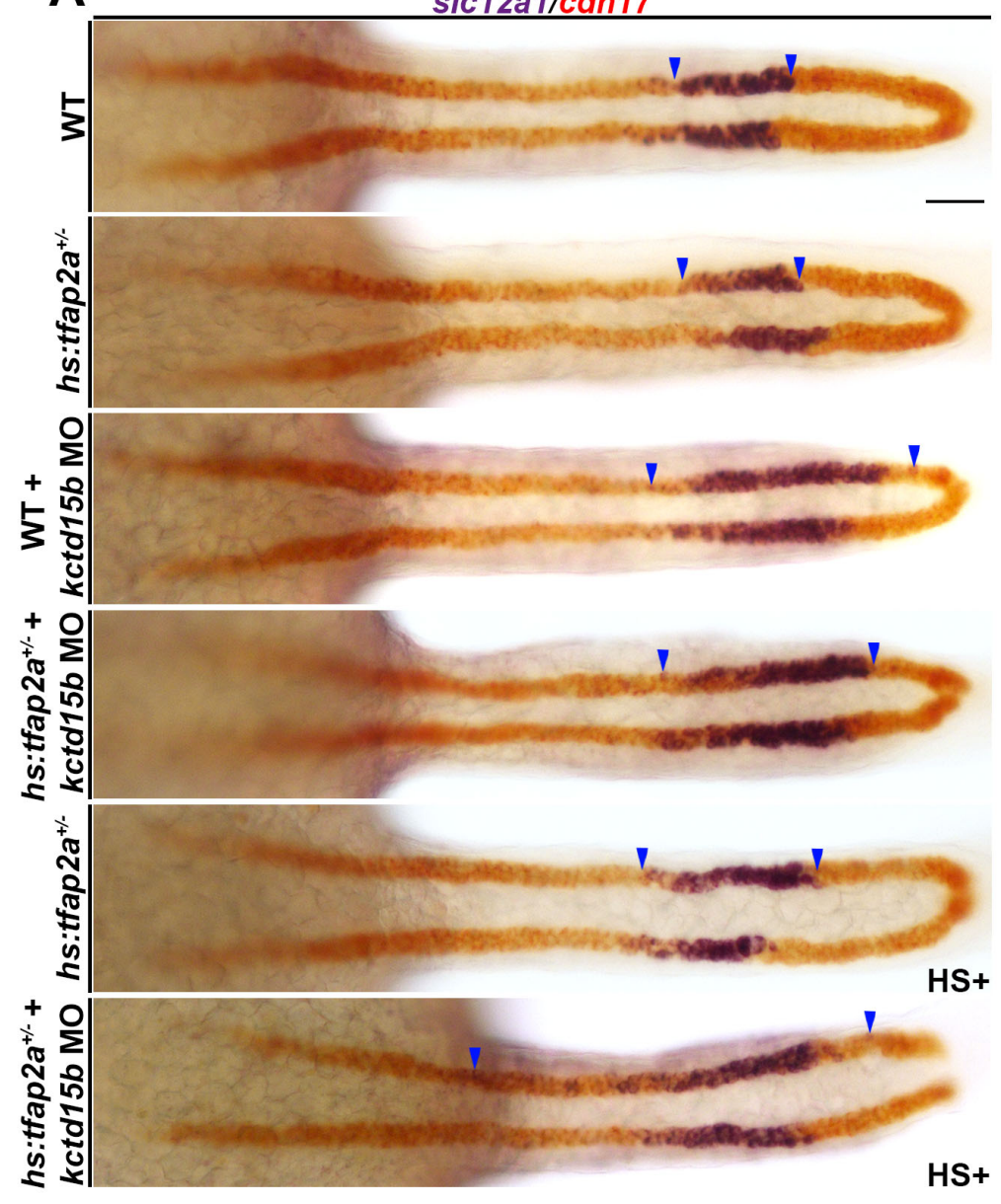

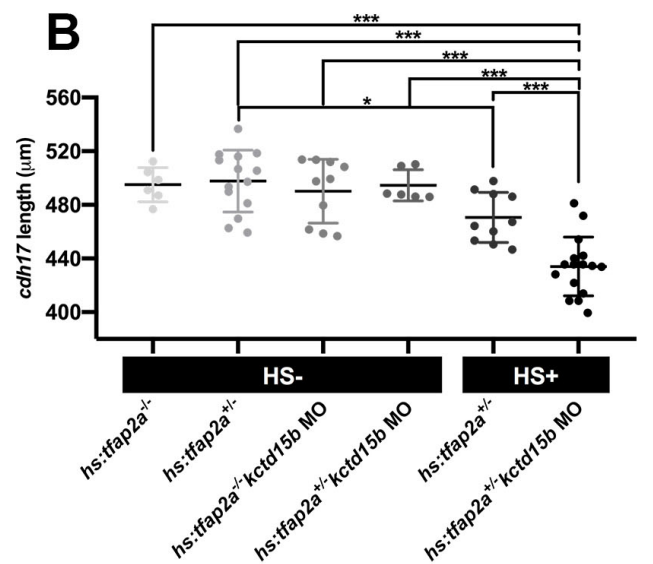

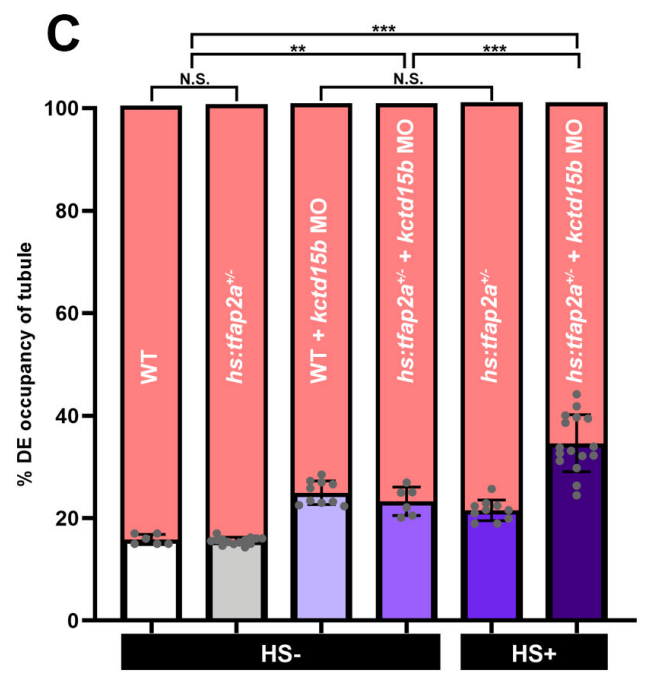


bioRxiv preprint doi: https://doi.org/10.1101/2020.01.17.910760; this version posted January 18,2020 . The copyright holder for this preprint (which was not certified by peer review) is the author/funder, who has granted bioRxiv a license to display the preprint in perpetuity. It is made available under aCC-BY-NC-ND 4.0 International license.

\section{Figure 8}
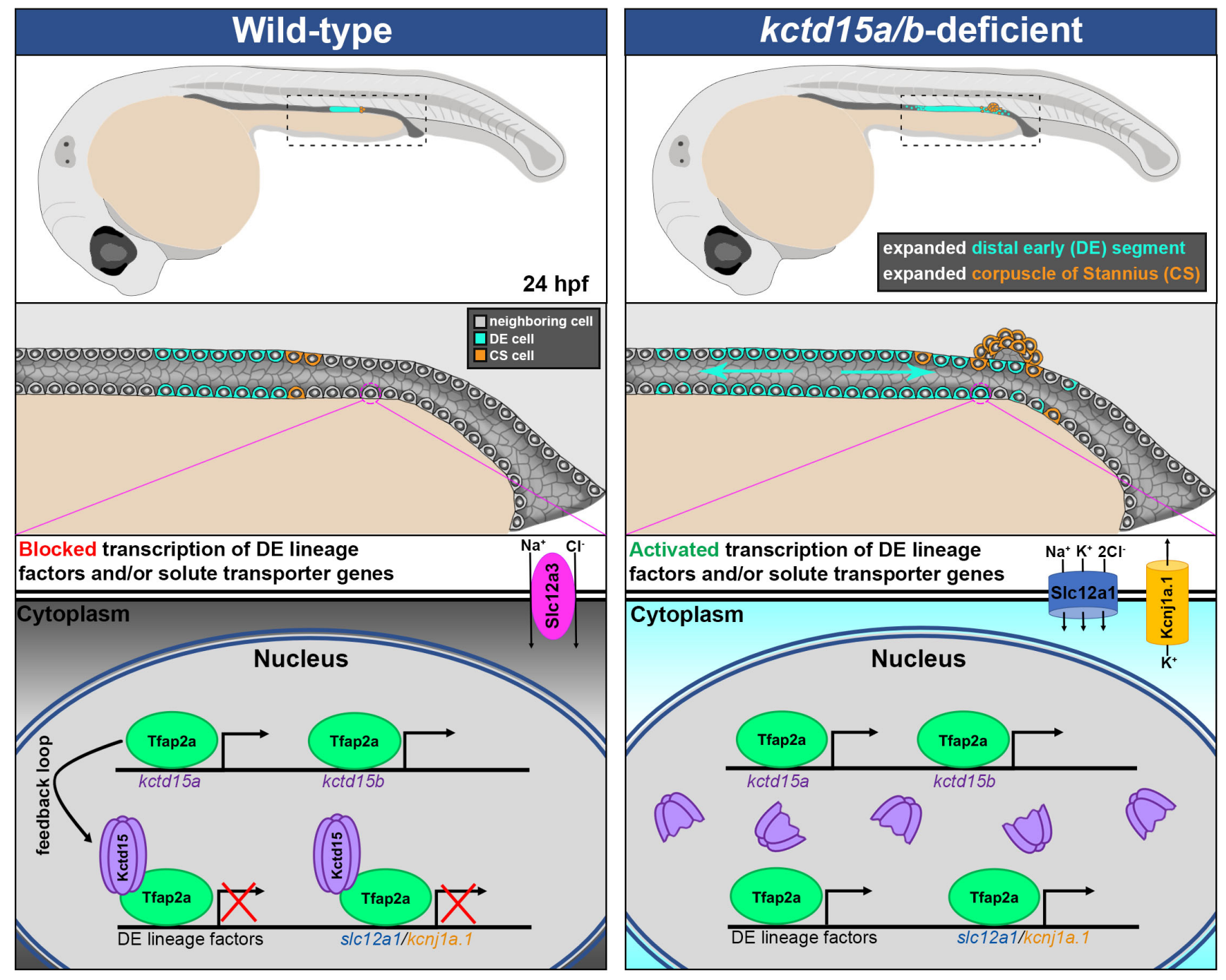


\section{Supplemental Materials}

Fig. S1 | Zebrafish Kctd15a and Kctd15b paralogs exhibit high amino acid sequence conservation with human KCTD15.

Fig. S2 | RT-PCR analysis confirms kctd15a and kctd15b MO reagents effectively block splicing.

Fig. S3 $\mid k c t d 15 a$ and $k c t d 15 b$ gRNAs edit the genome verifying successful crispant generation.

Fig. S4 | kctd15a overexpression represses ionocyte development.

Table S1 | Compilation of primer, morpholino oligonucleotide, and CRISPR sgRNA sequences. 
bioRxiv preprint doi: https://doi.org/10.1101/2020.01.17.910760; this version posted January 18, 2020. The copyright holder for this preprint (which was not certified by peer review) is the author/funder, who has granted bioRxiv a license to display the preprint in perpetuity. It is made available under aCC-BY-NC-ND 4.0 International license.

\section{Figure S1}

human KCTD15 V1 human KCTD15 V2 zebrafish Kctd15a zebrafish Kctd15b

human KCTD15 V1 human KCTD15 V2 zebrafish Kctd15a zebrafish Kctd15b

human KCTD15 V1 human KCTD15 V2 zebrafish Kctd15a zebrafish Kctd15b

human KCTD15 V1 human KCTD15 V2 zebrafish Kctd15a zebrafish Kctd15b

human KCTD15 V1 human KCTD15 V2 zebrafish Kctd15a zebrafish Kctd15b
1 MPHRKERPSGSSLHTHGSTGTAEGGNMSRLSLTRSPVSPLAAQGIPLPAQLTKSNAPVHI 1 MPHRKERPSGSSLHTHGSTGTAEGGNMSRLSLTRSPVSPLAAQGIPLPAQLTKSNAPVH I 1 ---------------------MSRLSLTRSPVSPLAAQGI PLPAQLTKSNAPVHI

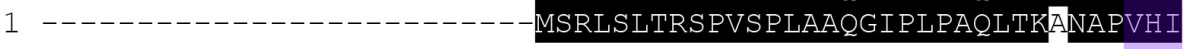

$\mathrm{BTB} / \mathrm{POZ}$ Domain

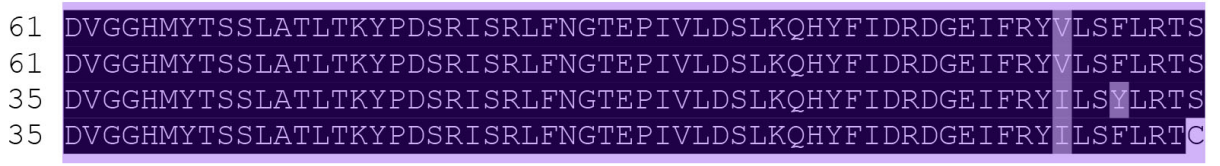

$\begin{aligned} 121 & \text { KLLLPDDFKDFSLLYEEARYYQLQPMVRELERWQQEQEQRRRSRACDCLVVRVTPDLGER } \\ 121 & \text { KLLLPDDFKDFSLLYEEARYYQLQPMVRELERWQEQEQRRRSRACDCLVVRVTPDLGER } \\ 95 & \text { KLLLPEDFKFLLLYEARYYQTPMVELERWKQEREQRRSAQPCECLVVRVTPDLGER } \\ 95 & \text { KLLLPDDFKDENLLYEEARYYQLSPMIKELERWKQEREQRRLANPCDCLVVRVTPDLGER }\end{aligned}$

181 IALSGEKALIEEVFPETGDVMCNSVNAGWNQDPTHVIRFPLNGYCRLNSVQDVIL------

181 IALSGEKALIEEVFPETGDVMCNSVNAGWNQDPTHVIRFPLNGYCRINSVQVLERLFQRC

155 IAVSGDKSLIEEIFPETGDVMCNSVNAGWNQDPTHVIRFPLNGYCRLNSVQVLERLFQKG

155 IALSGEKVLIEE IFPETGDVMCNSVNAGWNQDPTHVIRFPLNGYCRLNSVQVLERLFQKG

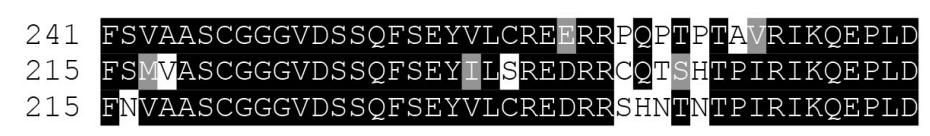


bioRxiv preprint doi: https://doi.org/10.1101/2020.01.17.910760; this version posted January 18, 2020. The copyright holder for this preprint (which was not certified by peer review) is the author/funder, who has granted bioRxiv a license to display the preprint in perpetuity. It is made

\section{Figure S2} available under aCC-BY-NC-ND 4.0 International license.

A

\section{kctd15a}
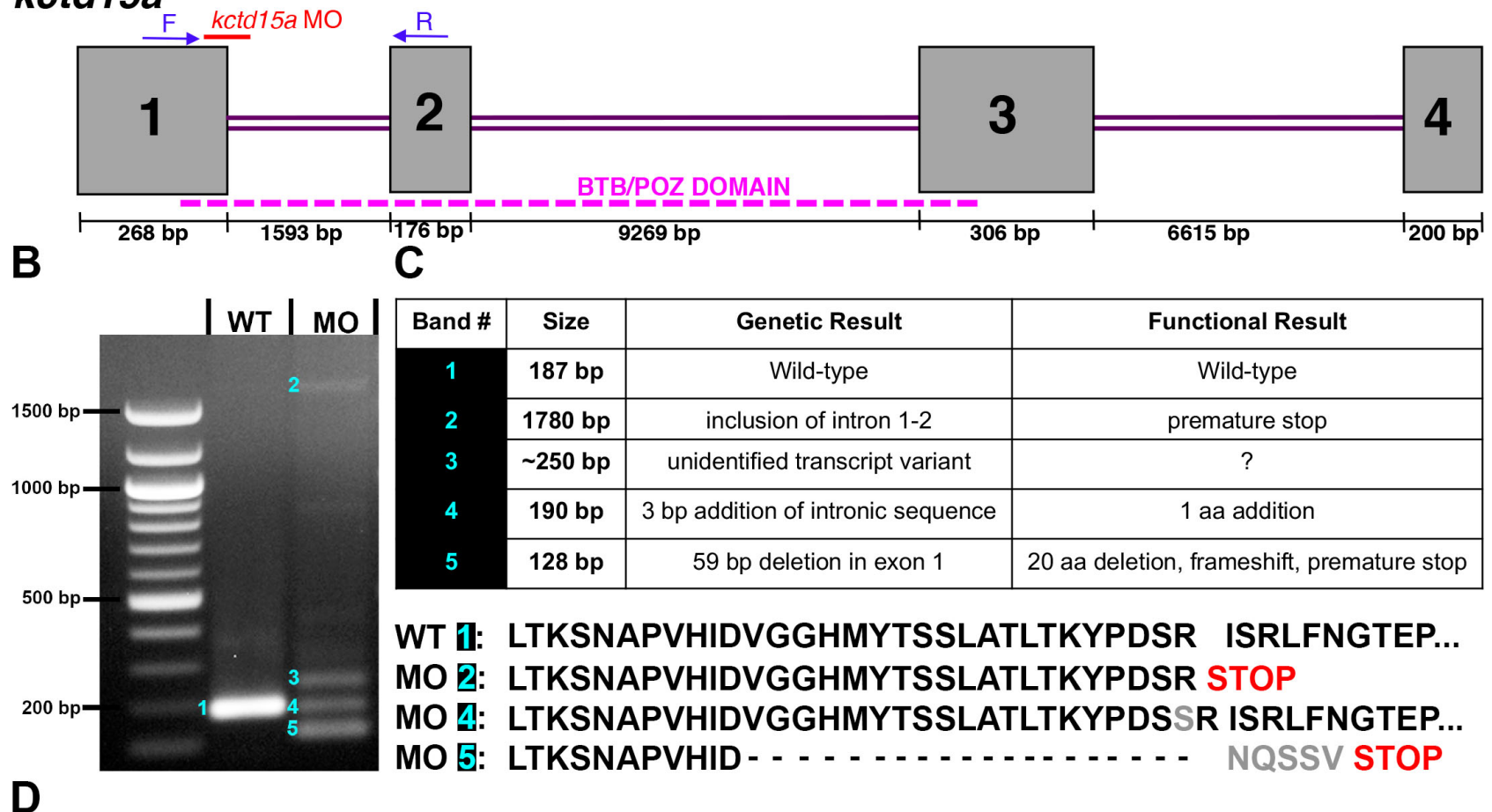

\begin{tabular}{|c|c|c|c|}
\hline Band \# & Size & Genetic Result & Functional Result \\
\hline 1 & $187 \mathrm{bp}$ & Wild-type & Wild-type \\
\hline 2 & $1780 \mathrm{bp}$ & inclusion of intron 1-2 & premature stop \\
\hline 3 & $\sim 250 \mathrm{bp}$ & unidentified transcript variant & $?$ \\
\hline 4 & $190 \mathrm{bp}$ & $3 \mathrm{bp}$ addition of intronic sequence & 1 aa addition \\
\hline 5 & $128 \mathrm{bp}$ & 59 bp deletion in exon 1 & 20 aa deletion, frameshift, premature stop \\
\hline
\end{tabular}

WT 1: LTKSNAPVHIDVGGHMYTSSLATLTKYPDSR ISRLFNGTEP...

MO 2: LTKSNAPVHIDVGGHMYTSSLATLTKYPDSR STOP

MO 4: LTKSNAPVHIDVGGHMYTSSLATLTKYPDSSR ISRLFNGTEP...

MO 5: LTKSNAPVHID - . - . . . . . . . . . . NQSSV STOP

\section{kctd15b}

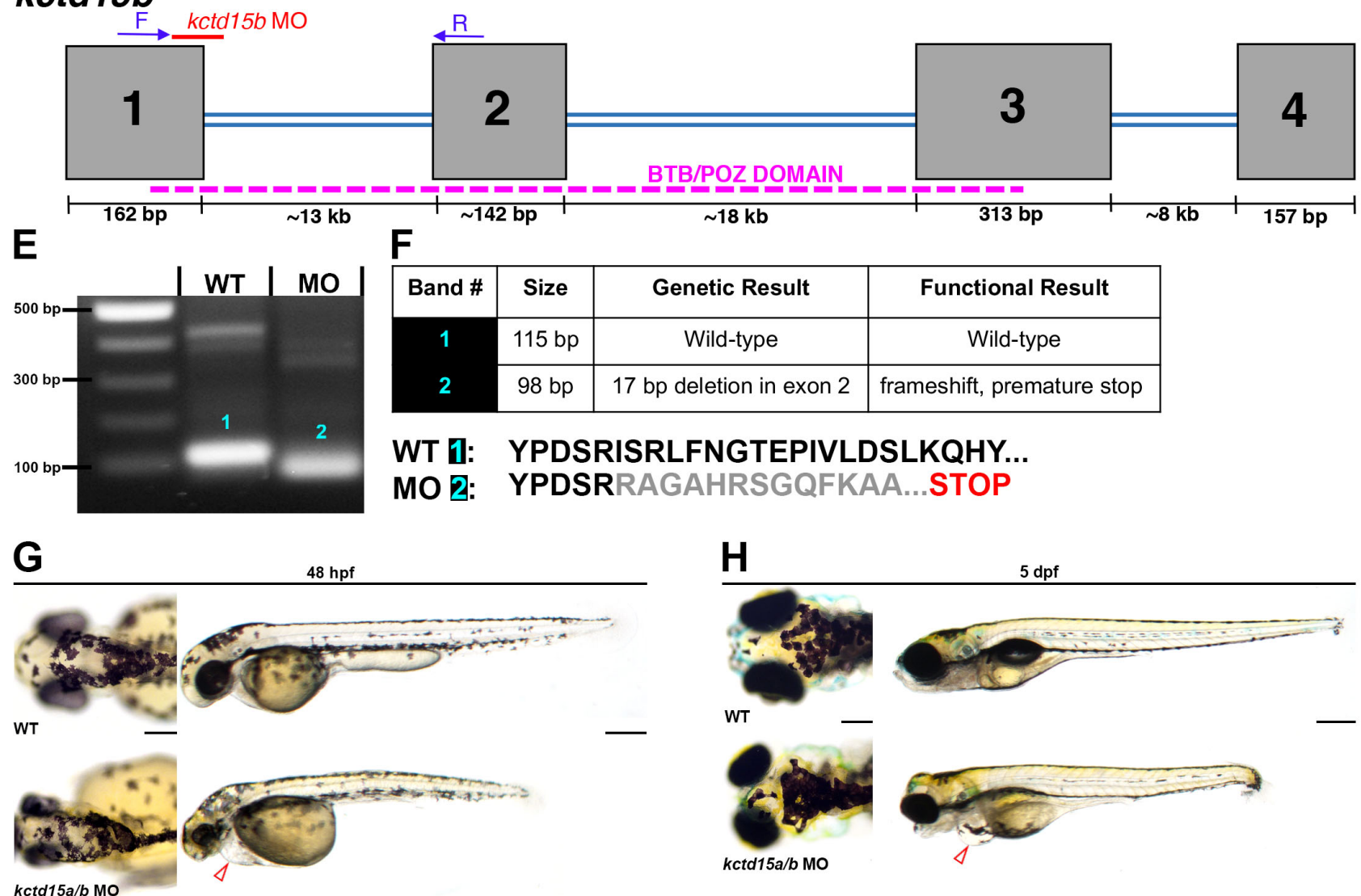


bioRxiv preprint doi: https://doi.org/10.1101/2020.01.17.910760; this version posted January 18, 2020. The copyright holder for this preprint (which was not certified by peer review) is the author/funder, who has granted bioRxiv a license to display the preprint in perpetuity. It is made

\section{Figure S3}

A

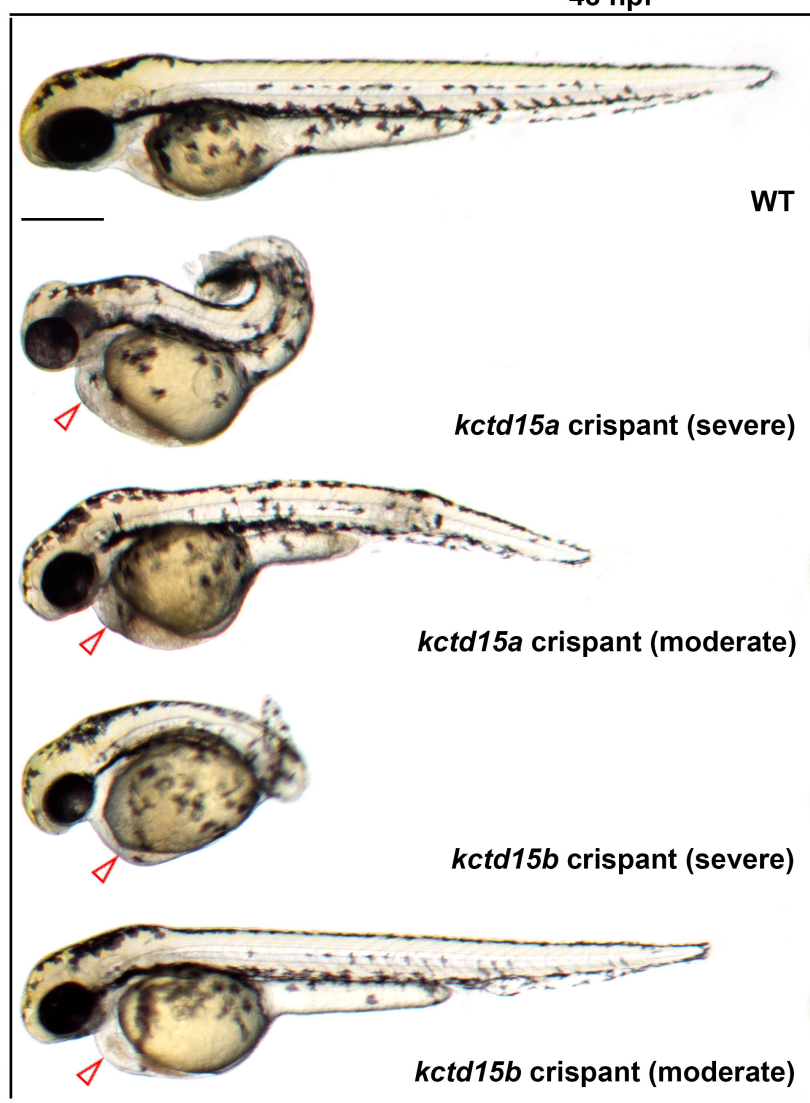

D

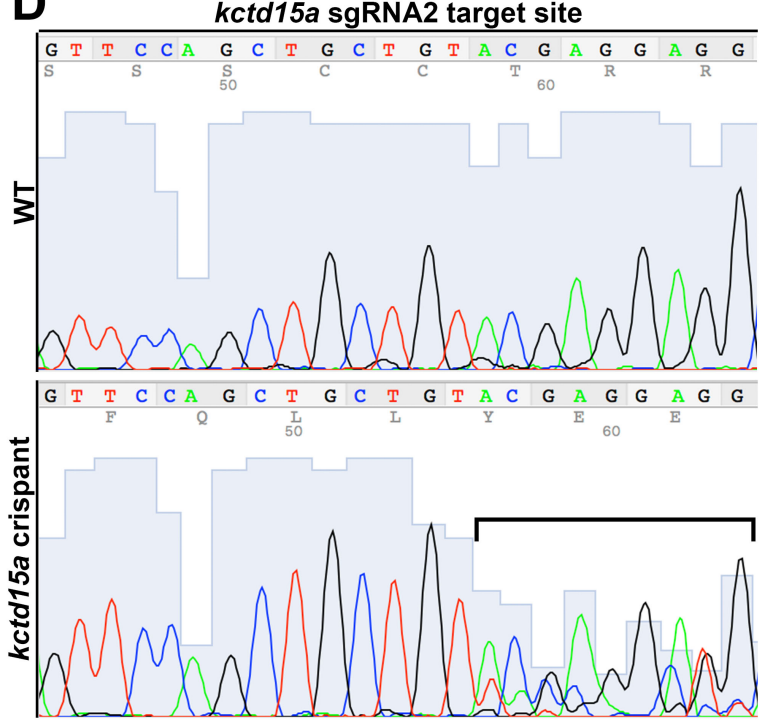

B

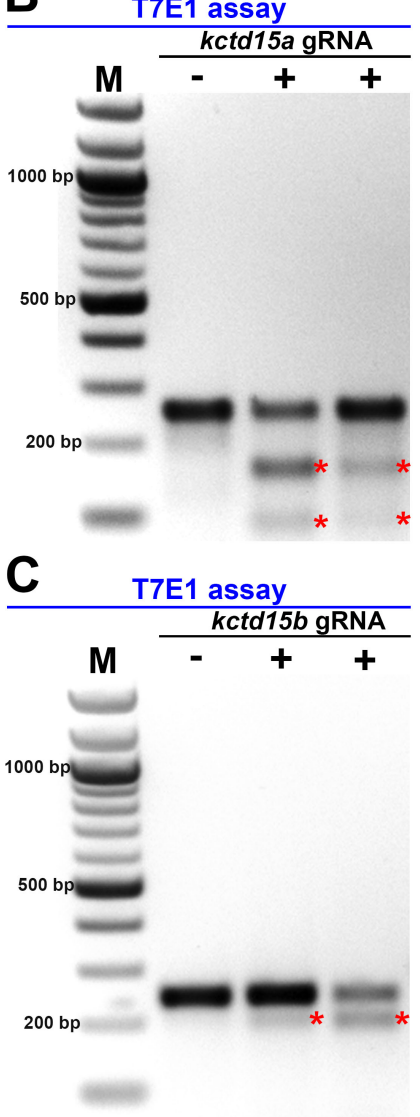

$\mathbf{E}$

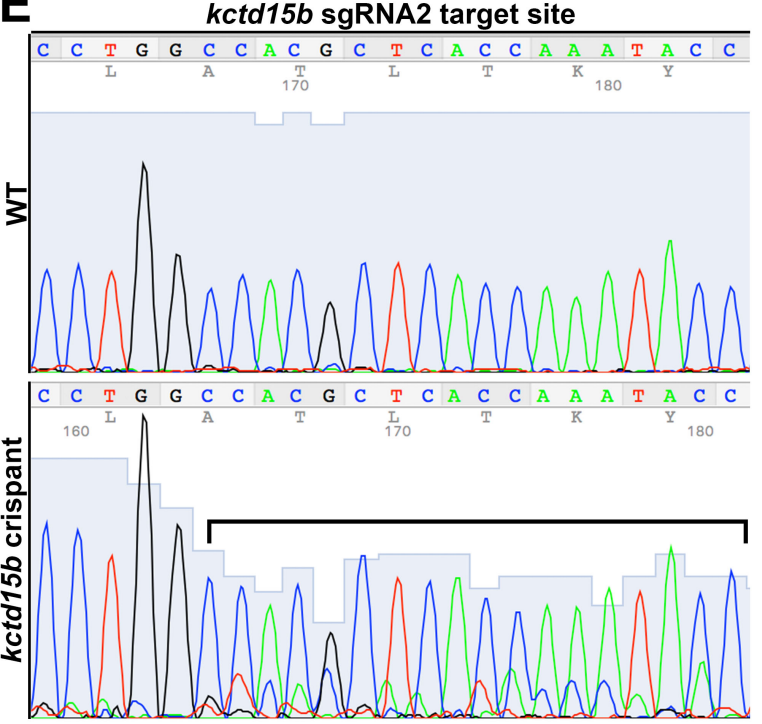

$\mathbf{F}$

\section{kctd15a}

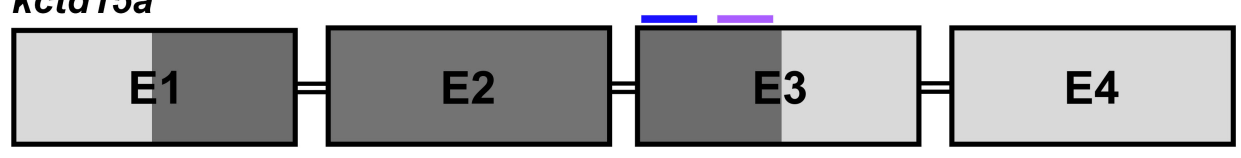

\section{kctd15b}

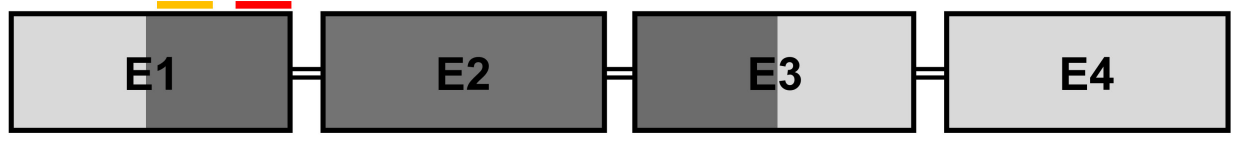

\begin{tabular}{|l|}
\hline KEY: \\
$\square$ BTB/POZ domain \\
$-\quad k c t d 15 a$ sgRNA1 \\
$-k c t d 15 a$ sgRNA2 \\
$-k c t d 15 b$ sgRNA1 \\
$-k c t d 15 b$ sgRNA2 \\
\hline
\end{tabular}


bioRxiv preprint doi: https://doi.org/10.1101/2020.01.17.910760; this version posted January 18,2020 . The copyright holder for this preprint (which was not certified by peer review) is the author/funder, who has granted bioRxiv a license to display the preprint in perpetuity. It is made

\section{Figure S4}

A

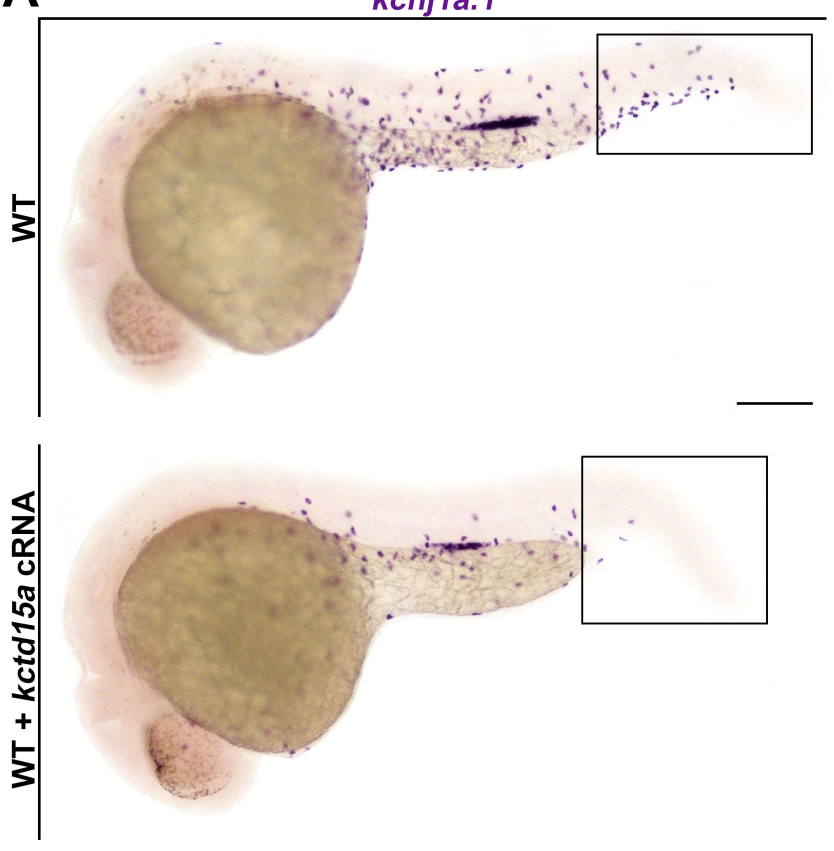

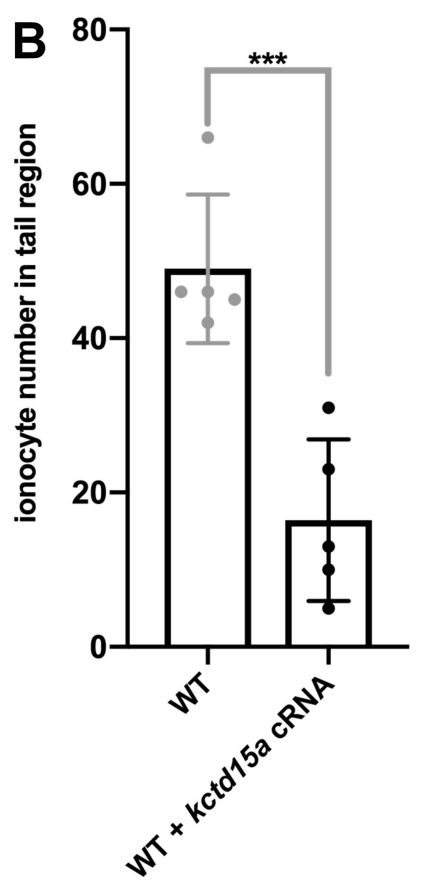


Table S1: Key Reagents

\begin{tabular}{|l|c|}
\hline \multicolumn{2}{|c|}{ morpholino oligonucleotides } \\
\hline kctd15a MO & 5'-CGAGTCATCTTACCTGGAGTCTGGG-3' \\
\hline kctd15b MO & 5'-CCAGACTCACGGTAAGACCTCTCAC-3' \\
\hline \multicolumn{2}{|c|}{ CRISPR sgRNAs } \\
\hline kctd15a sgRNA1 & GTTCCAGCTGCTGTACGAGG (-strand) \\
\hline kctd15a sgRNA2 & CTATTACCAGCTGACGCCCA (-strand) \\
\hline kctd15b sgRNA1 & TCCGCCCACGTCGATGTGCA (-strand) \\
\hline kctd15b sgRNA2 & GGTATTTGGTGAGCGTGGCC (-strand) \\
\hline & \multicolumn{1}{|c|}{ RT-PCR primers } \\
\hline kctd15a F & 5'-GCTCACTAAATCCAACGCTCCAGTCC-3' \\
\hline kctd15a R & 5'-GTAGCGGAATATCTCTCCATCTCGG-3' \\
\hline kctd15b F & 5'-ACATCGACGTGGGCGGACACATGTACACCA-3' \\
\hline kctd15b R & 5'-AAACTGTCCAGAACGATGGGCTCCGTCC-3' \\
\hline \multicolumn{2}{|c|}{ crispant genotyping primers } \\
\hline kctd15aCRISP F & 5'-ATGTTCATTCATTCATCCCTCC-3' \\
\hline kctd15aCRISP R & 5'-CTTGTCTCCACTGACAGCAATC-3' \\
\hline kctd15bCRISP F & 5'-CATCATTTTTCTCCATGGCTTT-3' \\
\hline kctd15bCRISP R & 5'-GGTGAGAGGTCTTACCGTGAGT-3' \\
\hline \multicolumn{2}{|c|}{ '-'tfap2a genotyping primers } \\
\hline hs:tfap2a F & 5'-CTCCTCTCAATGACAGCTG-3' \\
\hline hs:tfap2a R & 5-ATGGCGGTTGGAAGTCTGAA-3' \\
\hline
\end{tabular}

